\title{
Wave-vortex interaction in rotating shallow water. Part 1. One space dimension
}

\author{
By ALLEN C. KUO AND LORENZO M. POLVANI \\ Department of Applied Physics and Applied Mathematics, Columbia University, \\ New York, NY 10027, USA
}

(Received 1 December 1998 and in revised form 8 March 1999)

Using a physical space (i.e. non-modal) approach, we investigate interactions between fast inertio-gravity (IG) waves and slow balanced flows in a shallow rotating fluid. Specifically, we consider a train of IG waves impinging on a steady, exactly balanced vortex. For simplicity, the one-dimensional problem is studied first; the limitations of one-dimensionality are offset by the ability to define balance in an exact way. An asymptotic analysis of the problem in the small-amplitude limit is performed to demonstrate the existence of interactions. It is shown that these interactions are not confined to the modification of the wave field by the vortex but, more importantly, that the waves are able to alter in a non-trivial way the potential vorticity associated with that vortex. Interestingly, in this one-dimensional problem, once the waves have traversed the vortex region and have propagated away, the vortex exactly recovers its initial shape and thus bears no signature of the interaction. Furthermore, we prove this last result in the case of arbitrary vortex and wave amplitudes. Numerical integrations of the full one-dimensional shallow-water equations in strongly nonlinear regimes are also performed: they confirm that time-dependent interactions exist and increase with wave amplitude, while at the final state the vortex bears no sign of the interaction. In addition, they reveal that cyclonic vortices interact more strongly with the wave field than anticyclonic ones.

\section{Introduction}

Interactions between fast inertio-gravity (IG) waves and slow balanced motions, commonly referred to as 'wave-vortex' interaction, have been the subject of many recent studies. Whether such interactions exist, though, is still a partially open question. Dewar \& Killworth (1995) have reviewed some of the seemingly contradictory results in the current literature and have pointed out that some of the discrepancies may be due to fundamental differences between the various model equations that have been studied, while other discrepancies may simply be due to the use of different numerical schemes to solve the model equations.

While the question of whether and how IG waves interact with balanced motions is not fully resolved, most theoretical approaches to date have made use of modal decomposition and resonant wave interaction theory (Warn 1986; Yeh \& Dong 1988; Lelong \& Riley 1991; Vanneste \& Vial 1995; Bartello 1995; Majda \& Embid 1998). Related computational work (Errico 1984; Farge \& Sadourny 1989; Yuan \& Hamilton 1994; Dewar \& Killworth 1995) has relied heavily on these same modal decomposition tools. Such theoretical tools are rigorously applicable in the small Rossby or Froude number limit when the leading-order dynamics is linear. However, 
the modal decomposition approach is difficult to extend to the nonlinear regime and may also require assumptions on statistical homogeneity. Moreover, this approach provides no direct, physical picture of how IG waves may interact with slow balanced motions.

In this paper we attack the problem from a different angle. Rather than taking the eigenmodes of some linearized set of equations as the fundamental constituents of the interaction, we study actual physical objects. Specifically, we consider a train of IG waves impinging on an isolated, exactly balanced vortex. Because the vortex is initially steady, any changes to it can unequivocally be attributed to the IG waves: the extent to which the potential vorticity (PV) of the vortex is modified is the extent to which there exists a wave-vortex interaction. The results presented in this study are obtained in the context of the rotating $f$-plane shallow-water equations, the simplest model which permits both fast IG waves and slow balanced motions. By focusing on the physical evolution of the flow, we are able to directly pinpoint the time and location of the interaction and to observe and describe its physical characteristics. Moreover, our physical space approach is not limited to small Rossby or Froude number regimes and requires no assumptions of a statistical nature.

One study of wave-vortex interaction from a physical space viewpoint was carried out by Ford (1994) who showed how IG waves can be generated from a balanced flow. In this paper, we focus on the reverse problem, namely how IG waves originally external to a balanced flow are able modify it. Buhler \& McIntyre (1998) have recently considered a similar problem in a channel on a $\beta$-plane and have shown that IG waves forced at the boundary of the channel are able to irreversibly modify the interior PV gradient.

In this work, we restrict our attention to wave-vortex interactions in one spatial dimension. With this simplification, the notion of 'balance' can be defined unambiguously, facilitating the analysis of the problem. The more complex two-dimensional problem will be discussed in a future work.

The paper is divided up as follows. In $\S 2$ we set up the problem. An asymptotic analysis is performed $\S 3$, and the long-time interaction results are derived in $\S 4$. The asymptotic results are confirmed by numerical calculations in $\S 5$, where we examine the dependence of the interaction on the amplitudes of both the waves and the vortex, and the cyclonicity of the vortex. A brief discussion concludes the paper.

\section{Problem setup}

The rotating shallow-water equations (Pedlosky 1982), simplified by letting the time-dependent variables be functions of a single spatial coordinate, $x$, are in nondimensional form

$$
\left.\begin{array}{r}
\frac{\partial u}{\partial t}+u \frac{\partial u}{\partial x}+\frac{\partial \eta}{\partial x}-v=0 \\
\frac{\partial v}{\partial t}+u \frac{\partial v}{\partial x}+u=0 \\
\frac{\partial \eta}{\partial t}+\frac{\partial u}{\partial x}+\frac{\partial(\eta u)}{\partial x}=0
\end{array}\right\}
$$

where $u$ and $v$ represent $x$ and $y$ velocity components respectively, and $\eta$ represents the deviation of the free surface from a (dimensional) mean height $H$. The non-dimension- 
alization used here is standard (asterisks denote dimensional variables):

$$
h^{*}=H(1+\eta), \quad\left(u^{*}, v^{*}\right)=(\sqrt{g H})(u, v), \quad x^{*}=L_{d} x, \quad t^{*}=(1 / f) t,
$$

where $f$ is the Coriolis parameter, $g$ the gravitational acceleration and $L_{d} \equiv \sqrt{g H} / f$ is the Rossby deformation radius.

Although one-dimensional in space, the above shallow-water equations still contain both IG waves and balanced motions. These equations are thus the simplest starting point for studying wave-vortex interactions. Moreover, working with a single spatial dimension allows us to define balance in a unique way. Given the potential vorticity $\Pi$ of the flow, defined by

$$
\Pi(x, t) \equiv \frac{v_{x}(x, t)+1}{1+\eta(x, t)},
$$

one can invert for the balanced components of the flow $u_{b}, v_{b}$, and $\eta_{b}$, as follows. Geostrophic balance implies

$$
u_{b}=0 \quad \text { and } \quad v_{b}=\frac{\partial \eta_{b}}{\partial x} .
$$

Note that geostrophy is the only choice as a balance condition in one dimension; this follows from the fact that all spatially localized flows situated in an infinite domain that are not geostrophic will adjust to eventually become steady and geostrophic. In two dimensions, on the other hand, this is not necessarily true. Using (3) to eliminate $v$ from (2) yields an exact non-asymptotic potential vorticity inversion through the following second-order boundary value problem for $\eta_{b}$ :

$$
\frac{\partial^{2} \eta_{b}}{\partial x^{2}}-\Pi\left(1+\eta_{b}\right)+1=0
$$

together with appropriate evanescence boundary conditions. Thus the inversion consists in solving (4) for $\eta_{b}$, and then obtaining $v_{b}$ from (3). Of course, $u_{b}=0$.

To study wave-vortex interactions, we solve (1) with the initial conditions

$$
\boldsymbol{u}(x, t=0) \equiv\left(\begin{array}{c}
u(x, t=0) \\
v(x, t=0) \\
\eta(x, t=0)
\end{array}\right)=\boldsymbol{u}_{W}+\boldsymbol{u}_{V},
$$

where

$$
\boldsymbol{u}_{W}(x)=\left(\begin{array}{c}
u_{W}\left(x-x_{0}\right) \\
0 \\
0
\end{array}\right)
$$

and

$$
\boldsymbol{u}_{V}(x)=\left(\begin{array}{c}
0 \\
v_{V}(x) \\
\eta_{V}(x)
\end{array}\right) .
$$

As sketched in figure 1, the initial flow is divided into two distinct regions. Near $x=0$ we place a localized potential vorticity anomaly, which we will refer to as 

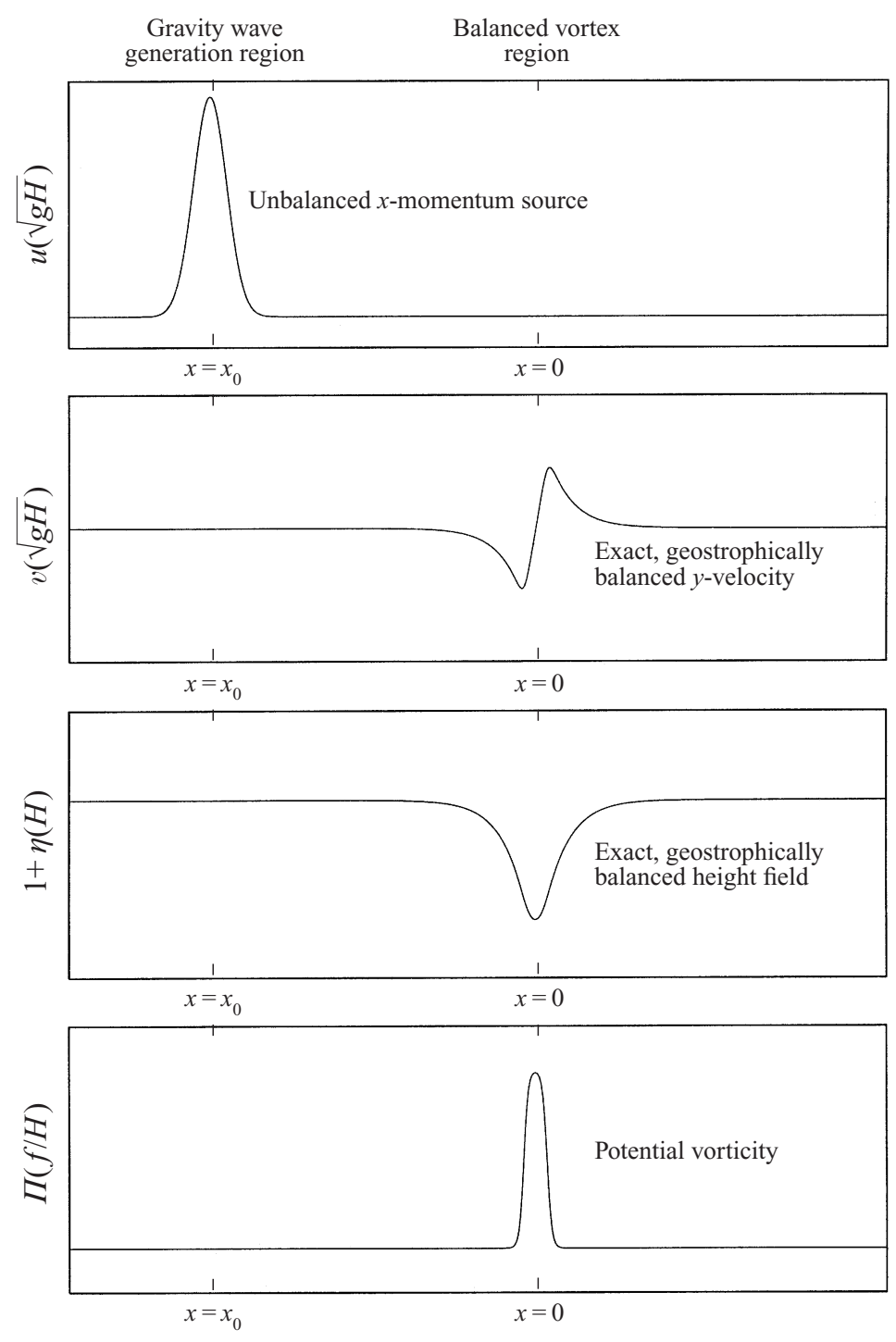

FIGURE 1. Initial conditions.

'the vortex' $\uparrow$ The corresponding fields $\boldsymbol{u}_{V}(x)$ are obtained from (3) and (4), and are thus in exact balance. Several deformation radii away, around $x=x_{0}$, we position an unbalanced, localized $x$-momentum anomaly $\boldsymbol{u}_{W}(x)$. A non-uniform $u(x)$ velocity implies that the flow is divergent, and thus near $x=x_{0}$ the flow will geostrophically adjust by radiating IG waves (Cahn 1945). These waves will eventually reach the vortex, and hence one may expect to see a wave-vortex interaction in the region near $x=0$.

It is fair to ask whether letting an unbalanced flow adjust is the only or perhaps best way to generate IG waves. An obvious alternative would be to use a wavemaker,

$\dagger$ This initial PV distribution in practice corresponds to two oppositely directed parallel jets. We use the term vortex in the sense of an object that is slow and balanced; the flow associated with this PV is actually infinitely slow and perfectly balanced at $t=0$. 
i.e. to specify the fields at some boundary so as to satisfy some linearized wave equation. The problem, of course, is how one specifies boundary conditions when the linearization assumption breaks down, an important regime we would like to explore in this paper. It is difficult to define general well-posed boundary conditions in the case of large wave amplitudes. This follows from the fact that the characteristics for (1) are not simple straight lines unless wave amplitudes are small (Kuo \& Polvani 1997).

To overcome these difficulties, we have thus opted for generating IG waves as a byproduct of a geostrophic adjustment. In the small-amplitude limit, we recover linear rotating gravity waves (Gill 1982). At large amplitudes, these motions can still be called IG waves, in the sense that they are fast and divergent; they no longer, however, obey simple dispersion relations, and may have complicated shapes including discontinuities (i.e. shocks, see Houghton 1969). Irrespective of amplitude, therefore, we will refer to the motions emanating from a geostrophic adjustment as IG waves.

\section{Asymptotic analysis}

We begin our analysis of the problem by constructing asymptotic solutions to (1) with initial conditions (5). This allows us to derive analytical expressions which describe precisely when, where, and how wave-vortex interaction occurs in the smallamplitude limit. The large-amplitude extensions are treated numerically in $\S 5$.

For purposes of deriving asymptotic solutions, we choose the following scalings for the initial conditions:

$$
\begin{gathered}
\boldsymbol{u}_{V}(x) \equiv \epsilon \hat{\boldsymbol{u}}_{V}(x), \\
\boldsymbol{u}_{W}(x) \equiv \epsilon^{2} \hat{\boldsymbol{u}}_{W}(x),
\end{gathered}
$$

where $\epsilon \ll 1$. Other scalings are of course possible, but (8) and (9) are chosen because they provide the clearest possible scenario for wave-vortex interactions. Specifically, (8) eliminates the well-known vortex effect on waves via refraction (see Buhler \& McIntyre 1998), while (9) pushes unwanted wave-wave effects (i.e. wave steepening) one order beyond the wave effects on the vortex, which are ultimately the main focus of this paper. The above scalings therefore clearly separate the exactly balanced vortex at lowest order, the IG waves arising solely from the unbalanced region at next order, and finally the nonlinear interaction of the two at third order.

\subsection{Analysis of the field variables}

Expanding the solution, as is customary, in the form

$$
\boldsymbol{u}(x, t) \equiv \epsilon \boldsymbol{u}^{(1)}(x, t)+\epsilon^{2} \boldsymbol{u}^{(2)}(x, t)+\epsilon^{3} \boldsymbol{u}^{(3)}(x, t)+O\left(\epsilon^{4}\right)
$$

and substituting into (1), yields the following sequence of problems.

At $O(\epsilon)$

$$
\frac{\partial u^{(1)}}{\partial t}+\frac{\partial \eta^{(1)}}{\partial x}-v^{(1)}=0, \quad \frac{\partial v^{(1)}}{\partial t}+u^{(1)}=0, \quad \frac{\partial \eta^{(1)}}{\partial t}+\frac{\partial u^{(1)}}{\partial x}=0,
$$

with initial conditions

$$
u^{(1)}(x, t=0)=0, \quad v^{(1)}(x, t=0)=\hat{v}_{V}(x), \quad \eta^{(1)}(x, t=0)=\hat{\eta}_{V}(x) .
$$


Since $\hat{v}_{V}$ and $\hat{\eta}_{V}$ are already in geostrophic balance, it immediately follows that

$$
\begin{gathered}
u^{(1)}(x, t)=0, \\
v^{(1)}(x, t)=\hat{v}_{V}(x), \\
\eta^{(1)}(x, t)=\hat{\eta}_{V}(x) .
\end{gathered}
$$

Hence $\boldsymbol{u}^{(1)}$ is simply the exactly balanced time-independent vortex.

At $O\left(\epsilon^{2}\right)$

$$
\frac{\partial u^{(2)}}{\partial t}+\frac{\partial \eta^{(2)}}{\partial x}-v^{(2)}=0, \quad \frac{\partial v^{(2)}}{\partial t}+u^{(2)}=0, \quad \frac{\partial \eta^{(2)}}{\partial t}+\frac{\partial u^{(2)}}{\partial x}=0,
$$

with initial conditions

$$
u^{(2)}(x, t=0)=\hat{u}_{W}\left(x-x_{0}\right), \quad v^{(2)}(x, t=0)=0, \quad \eta^{(2)}(x, t=0)=0 .
$$

The solution at this order is found, following Gill (1976), by eliminating $v^{(2)}$ and $\eta^{(2)}$ in (16) to yield a single homogeneous dispersive wave equation for $u^{(2)}$

$$
\frac{\partial^{2} u^{(2)}}{\partial t^{2}}-\frac{\partial^{2} u^{(2)}}{\partial x^{2}}+u^{(2)}=0 .
$$

Once $u^{(2)}$ is obtained via Fourier transforms, the solution for $v^{(2)}$ and $\eta^{(2)}$ can be found in terms of it. The complete $O\left(\epsilon^{2}\right)$ solution is

$$
\begin{gathered}
u^{(2)}(x, t)=\frac{1}{2 \sqrt{2 \pi}} \int_{-\infty}^{\infty} \hat{U}_{W}(k)\left(\mathrm{e}^{-\mathrm{i}\left[k x+\sqrt{k^{2}+1} t\right]}+\mathrm{e}^{-\mathrm{i}\left[k x-\sqrt{k^{2}+1} t\right]}\right) \mathrm{d} k, \\
v^{(2)}(x, t)=-\int_{0}^{t} u^{(2)}(x, s) \mathrm{d} s, \\
\eta^{(2)}(x, t)=-\int_{0}^{t} \frac{\partial u^{(2)}(x, s)}{\partial x} \mathrm{~d} s,
\end{gathered}
$$

where $\hat{U}_{W}(k)$ is the spatial Fourier transform of the initial condition

$$
\hat{U}_{W}(k)=\frac{1}{\sqrt{2 \pi}} \int_{-\infty}^{\infty} \hat{u}_{W}\left(x-x_{0}\right) \mathrm{e}^{\mathrm{i} k x} \mathrm{~d} x
$$

At this order the solution is identical to that obtained from a linear, time-dependent geostrophic adjustment problem (Cahn 1945). Therefore, we refer to $\boldsymbol{u}^{(2)}(x, t)$ as pure IG waves because they originate solely from the unbalanced region near $x=x_{0}$ and are independent of the vortex part of the solution $\boldsymbol{u}^{(1)}$. If the analysis were to stop at this order, one would conclude that no wave-vortex interaction exists.

However, at $O\left(\epsilon^{3}\right)$

$$
\begin{aligned}
\frac{\partial u^{(3)}}{\partial t}+\frac{\partial \eta^{(3)}}{\partial x}-v^{(3)}=0, \quad \frac{\partial v^{(3)}}{\partial t}+u^{(3)}=-u^{(2)} \frac{\partial v^{(1)}}{\partial x} & \\
& \frac{\partial \eta^{(3)}}{\partial t}+\frac{\partial u^{(3)}}{\partial x}=-\frac{\partial\left(\eta^{(1)} u^{(2)}\right)}{\partial x} .
\end{aligned}
$$

with initial conditions

$$
u^{(3)}(x, t=0)=0, \quad v^{(3)}(x, t=0)=0, \quad \eta^{(3)}(x, t=0)=0 .
$$

Again, eliminating $v^{(3)}$ and $\eta^{(3)}$ from (23) yields a single inhomogeneous dispersive 
wave equation for $u^{(3)}$,

$$
\frac{\partial^{2} u^{(3)}}{\partial t^{2}}-\frac{\partial^{2} u^{(3)}}{\partial x^{2}}+u^{(3)}=\mathscr{I}_{W V}(x, t)
$$

where the inhomogeneous term $\mathscr{I}_{W V}(x, t)$ is given by

$$
\mathscr{I}_{W V}=-u^{(2)} \frac{\partial v^{(1)}}{\partial x}+\frac{\partial^{2}\left(\eta^{(1)} u^{(2)}\right)}{\partial x^{2}} .
$$

This source term (26) contains products of $\boldsymbol{u}^{(1)}$ (the vortex) and $\boldsymbol{u}^{(2)}$ (the IG waves), and therefore can only be interpreted as a wave-vortex interaction term. The interaction, as (25) shows, is essential to generating the third-order solution, since $\mathscr{I}_{W V}$ becomes non-zero only when the IG waves enter the vortex region near $x=0$. We solve (25) using Duhamel's principle (John 1978), and thus obtain the complete $O\left(\epsilon^{3}\right)$ solution

$$
\begin{gathered}
u^{(3)}(x, t)=\frac{1}{2 \sqrt{2 \pi}} \int_{0}^{t}\left[\int_{-\infty}^{\infty} \frac{\mathrm{i} \hat{\mathscr{I}}_{W V}(k, s)}{\sqrt{k^{2}+1}}\left(\mathrm{e}^{-\mathrm{i}\left[k x+\sqrt{k^{2}+1}(t-s)\right]}-\mathrm{e}^{-\mathrm{i}\left[k x-\sqrt{k^{2}+1}(t-s)\right]}\right) \mathrm{d} k\right] \mathrm{d} s, \\
v^{(3)}(x, t)=-\int_{0}^{t}\left(u^{(3)}(x, s)+u^{(2)}(x, s) \frac{\partial \hat{v}_{V}}{\partial x}\right) \mathrm{d} s, \\
\eta^{(3)}(x, t)=-\int_{0}^{t}\left(\frac{\partial u^{(3)}(x, s)}{\partial x}+\frac{\partial\left(\hat{\eta}_{v} u^{(2)}(x, s)\right)}{\partial x}\right) \mathrm{d} s,
\end{gathered}
$$

where $\hat{\mathscr{I}}_{W V}$ is the spatial Fourier transform of $\mathscr{I}_{W V}$.

To sum up the asymptotic analysis: since the lowest-order solution $\boldsymbol{u}^{(1)}$ consists of a balanced vortex only, and the next-order solution $\boldsymbol{u}^{(2)}$ consists of IG waves only, we conclude that the generation of $\boldsymbol{u}^{(3)}$ by the previous two establishes the existence of a 'wave-vortex' interaction.

The question, at this point, is whether the IG waves modify the vortex, vice versa, or both. In other words, how do we interpret $\boldsymbol{u}^{(3)}$ ? Considering the fact that the Poincare wave operator appears on the left-hand side of (25), one might argue that $\boldsymbol{u}^{(3)}$ is simply IG waves. However, the same operator appears at all orders of perturbation beyond lowest order. Almost by definition then, since the vortex solution at lowest order is time independent, one would be forced to conclude that the vortex modifies the IG waves but not vice versa.

However, from (23), it is unclear whether the $\boldsymbol{u}^{(3)}$ solution contains only IG waves: a balanced component could be present, in which case we would have to conclude that IG waves affect the vortex as well. It turns out that this conclusion is the correct one, and is revealed through an analysis of the potential vorticity.

\subsection{Analysis of the potential vorticity}

Substituting the formal ansatz (10) into the definition (2) implies the following expression for the potential vorticity $\Pi(x, t)$ :

$$
\Pi(x, t)=\Pi^{(0)}+\epsilon \Pi^{(1)}+\epsilon^{2} \Pi^{(2)}+\epsilon^{3} \Pi^{(3)}+O\left(\epsilon^{4}\right),
$$

where

$$
\Pi^{(0)}=1
$$




$$
\begin{gathered}
\Pi^{(1)}=v_{x}^{(1)}(x)-\eta^{(1)}(x), \\
\Pi^{(2)}=-\eta^{(1)}(x) \Pi^{(1)}+v_{x}^{(2)}(x, t)-\eta^{(2)}(x, t), \\
\Pi^{(3)}=\left(\left(\eta^{(1)}(x)\right)^{2}-\eta^{(2)}(x, t)\right) \Pi^{(1)}+v_{x}^{(3)}(x, t)-\eta^{(3)}(x, t) .
\end{gathered}
$$

$\Pi^{(0)}$, the lowest order PV, is simply the background value $f / H$ properly non-dimensionalized. $\Pi^{(1)}$ is the linearized PV associated with the $\boldsymbol{u}^{(1)}$ solution (the initial vortex), and is time independent. $\Pi^{(2)}$ appears to be time dependent, but it is not. Since (16) implies

$$
\frac{\partial\left(v_{x}^{(2)}-\eta^{(2)}\right)}{\partial t}=0
$$

and since $\left(v_{x}^{(2)}-\eta^{(2)}\right)$ vanishes at $t=0$, the expression for $\Pi^{(2)}$ reduces to

$$
\Pi^{(2)}=-\eta^{(1)}(x) \Pi^{(1)},
$$

which explicitly shows the time independence. The $\boldsymbol{u}^{(2)}$ fields are just linear IG waves and hence, as is commonly believed, 'carry' no PV. To $O\left(\epsilon^{2}\right)$ therefore, the PV is time independent and is solely associated with the initial vortex.

The interesting result is seen at $O\left(\epsilon^{3}\right)$. We can show explicitly that $\Pi^{(3)}$ is time dependent, and therefore the $\boldsymbol{u}^{(3)}$ fields do in fact 'carry' PV. From (23), one may derive an expression for $\left(v_{x}^{(3)}-\eta^{(3)}\right)$ in terms of the $O\left(\epsilon^{2}\right)$ variables. Substituting that expression into (34) yields a simpler expression for $\Pi^{(3)}$ :

$$
\Pi^{(3)}=\eta^{(1)}(x)^{2} \Pi^{(1)}+v^{(2)}(x, t) \Pi_{x}^{(1)}
$$

which is explicitly time dependent, and thus demonstrates that the IG waves can affect the vortex.

Alternatively, one may deduce this fact by examining the equation for the evolution of PV:

$$
\frac{\partial}{\partial t} \Pi(x, t)+u \frac{\partial}{\partial x} \Pi(x, t)=0,
$$

which up to $O\left(\epsilon^{2}\right)$ yields

$$
\frac{\partial}{\partial t} \Pi^{(0)}=\frac{\partial}{\partial t} \Pi^{(1)}=\frac{\partial}{\partial t} \Pi^{(2)}=0
$$

but at $O\left(\epsilon^{3}\right)$ yields

$$
\frac{\partial}{\partial t} \Pi^{(3)}=-u^{(2)} \frac{\partial}{\partial x} \Pi^{(1)}
$$

This evolution equation shows how the nonlinear coupling of the PV associated with the vortex, $\Pi^{(1)}$, and the velocity field due to the incoming IG waves, $u^{(2)}$, produces changes in the PV, which given our scaling choices (8)-(9), occurs only at $O\left(\epsilon^{3}\right)$ here.

\subsection{Analysis of the energy}

The key effect of waves on the vortex is described by equation (40). In this subsection, for completeness, we provide a further description of the interaction in terms of the energy exhange between the waves and the vortex. The total energy is defined by

$$
E=\frac{1}{2}\left(u^{2}+v^{2}\right)(1+\eta)+\frac{1}{2}(1+\eta)^{2},
$$


and it obeys the conservation law (Whitham 1974)

$$
\frac{\partial E}{\partial t}+\frac{\partial}{\partial x}\left[\frac{1}{2}\left(u^{3}+u v^{2}\right)(1+\eta)+u(1+\eta)^{2}\right]=0 .
$$

Substituting (10) into (42) and collecting terms at different orders in $\epsilon$ yields equations at even powers of epsilon (the odd powers yield trivial identities through $O\left(\epsilon^{5}\right)$ ).

At $O\left(\epsilon^{2}\right)$ one obtains

$$
\frac{\partial E^{(1)}}{\partial t}=0
$$

where $E^{(1)} \equiv \frac{1}{2}\left(u^{(1)^{2}}+v^{(1)^{2}}\right)+\frac{1}{2} \eta^{(1)^{2}}$. At lowest order, the energy $\dagger$ is only associated with the vortex and, since the vortex is balanced, the energy is time independent.

At $O\left(\epsilon^{4}\right)$, the energy equation takes the form

$$
\frac{\partial E^{(2)}}{\partial t}+\frac{\partial\left(u^{(2)} \eta^{(2)}\right)}{\partial x}=0
$$

where $E^{(2)} \equiv \frac{1}{2}\left(u^{(2)^{2}}+v^{(2)^{2}}\right)+\frac{1}{2} \eta^{(2)^{2}}$. This equation, identical to the one found in Gill (1982), reflects the simple fact that, at this order, the time change in energy in a region is solely due to the IG wave flux in and out of that region. Thus, at this order, there is no interaction between the IG waves and the vortex.

Finally, at $O\left(\epsilon^{6}\right)$, one obtains the energy equation associated with the third-order solution:

$$
\frac{\partial E^{(3)}}{\partial t}+\frac{\partial\left(u^{(3)} \eta^{(3)}\right)}{\partial x}=-v^{(3)} u^{(2)} \frac{\partial v^{(1)}}{\partial x}-\eta^{(3)} \frac{\partial\left(\eta^{(1)} u^{(2)}\right)}{\partial x},
$$

where $E^{(3)}=\frac{1}{2}\left(u^{(3)^{2}}+v^{(3)^{2}}\right)+\frac{1}{2} \eta^{(3)^{2}}$. This energy equation differs from the previous two. At this order, $E^{(3)}$ changes either because of a flux or due to an interaction of the first- and second-order solutions (cf. the right-hand side of (45), remembering that the $O\left(\epsilon^{3}\right)$ solution is created solely from the interaction of the first- and second-order solutions). In other words, $E^{(3)}$ is created or destroyed when the IG waves come in contact with the initially balanced vortex. Hence, at this order, there is an energy exchange between waves and vortices.

\subsection{An illustrative example of wave-vortex interaction}

We now graphically illustrate this wave-vortex interaction by plotting, for a specific initial condition, the asymptotic solutions given in $\S 3.1$ and $\S 3.2$. As already mentioned, the initial condition is composed of an exactly balanced vortex part, $\boldsymbol{u}_{V}=\epsilon \hat{\boldsymbol{u}}_{V}$, and an unbalanced part, $\boldsymbol{u}_{W}=\epsilon^{2} \hat{\boldsymbol{u}}_{W}$, which generates IG waves (see figure 1 for a schematic view).

The vortex part is specified initially as the solution of

$$
\begin{gathered}
\frac{\mathrm{d}^{2} \hat{\eta}_{V}}{\mathrm{~d} x^{2}}-\hat{\eta}_{V}=\frac{1}{2}\left(\tanh \left[\frac{\left(x+\frac{1}{2}\right)}{S_{V}}\right]+\tanh \left[\frac{\left(-x+\frac{1}{2}\right)}{S_{V}}\right]\right), \\
\hat{v}_{V}=\frac{\mathrm{d} \hat{\eta}_{V}}{\mathrm{~d} x}, \\
\hat{u}_{V}=0 .
\end{gathered}
$$

$\dagger$ Note that the quantities $E^{(i)}$, as defined above at each order, are not the coefficients of the powers of $\epsilon$ that appear upon substituting (10) into (41). 
These initial conditions on $\boldsymbol{u}_{V}$ correspond to a positive tanh 'bump' in the potential vorticity field, roughly one deformation radius wide. The two equations (46) and (47) represent the exact balance procedure given in $\S 2$ at $O(\epsilon)$. The steepness of the edges of this bump is controlled by the parameter $S_{V}$. For illustrative purposes, we choose $S_{V}=0.2$.

For the unbalanced component, we specify

$$
\hat{u}_{W}(x)=\mathrm{e}^{-\left(x-x_{0}\right)^{2}}, \quad \hat{v}_{W}(x)=0, \quad \hat{\eta}_{W}(x)=0 .
$$

This unbalanced component of the initial conditions will cause IG waves to be generated from the region near $x=x_{0}$. We choose $x_{0}$ large enough so that the unbalanced region is well separated from the vortex at $t=0$. For the results presented in this paper we have set $x_{0}=-14$.

The asymptotic solution is plotted in figure 2. The left column shows the height field at five different times and the right column shows the corresponding PV. In each frame, the first three orders of perturbation are plotted. For clarity, the curves for the height field have been offset from their zero mean.

Consider first the height fields. At $t=10$ the exactly balanced initial vortex (dashed dotted line) and the linear IG wave train (dashed line) impinging on it from the left, though close, have not yet interacted. The third-order solution (solid line) is therefore identically zero. By $t=15$ the IG waves have reached the vortex and their interaction has generated the third-order solution. At $t=25$, the IG waves are seen leaving the vortex region, and the third-order solution resulting from the wave-vortex interaction is composed of transmitted waves (relatively strong) and reflected waves (relatively weak). Hence it is clear that the presence of the vortex modifies the total IG wave field. Such effects of the balanced motion on IG waves is well known (see for instance Dewar \& Killworth 1995 or Buhler \& McIntyre 1998) but in this case, the effect is purely nonlinear rather than due to refraction or linear reflection.

Note most importantly that the IG waves affect the vortex as well. To see this, consider the evolution of the potential vorticity in figure 2 . As already mentioned, the PV is time independent at first and second order; we plot these for reference. The effect of waves on the PV appears at $O\left(\epsilon^{3}\right)$. Notice that $\Pi^{(3)}$ becomes time dependent only around $t=15$, after the IG waves have entered the vortex region, thus demonstrating explicitly that IG waves alter the vortex.

It is important to remark that the appearance of wave-vortex interactions at $O\left(\epsilon^{3}\right)$ in this analysis is not a fundamental property of the interaction. It is simply an artifact of our scaling choices (8) and (9), which are guided by our interest in isolating wave-vortex interactions from wave-wave interactions. The latter are easily treated by standard perturbation techniques (Kevorkian \& Cole 1981). Our scaling choices simply relegate wave-wave interaction to $O\left(\epsilon^{4}\right)$. It is easy to show that the alternative scaling choice $\boldsymbol{u}_{V} \sim \boldsymbol{u}_{W} \sim \epsilon$ leads to a conventional advective Rossby number expansion of the shallow-water equations, in which case $\epsilon$ can be interpreted as a Rossby number. With that more conventional scaling, wave-vortex interactions would appear at $O\left(\epsilon^{2}\right)$ (together with wave-wave interactions). It would then follow that, at small Rossby number, wave-vortex interactions scale like the square of the Rossby number.

\section{The limit $t \rightarrow \infty$}

Having described how IG waves interact with a balanced vortex as they impinge upon it, we now turn our attention to the question of whether this interaction leaves a long-time signature on the vortex after the IG waves have propagated away from 
Wave-vortex interaction in rotating shallow water. Part 1
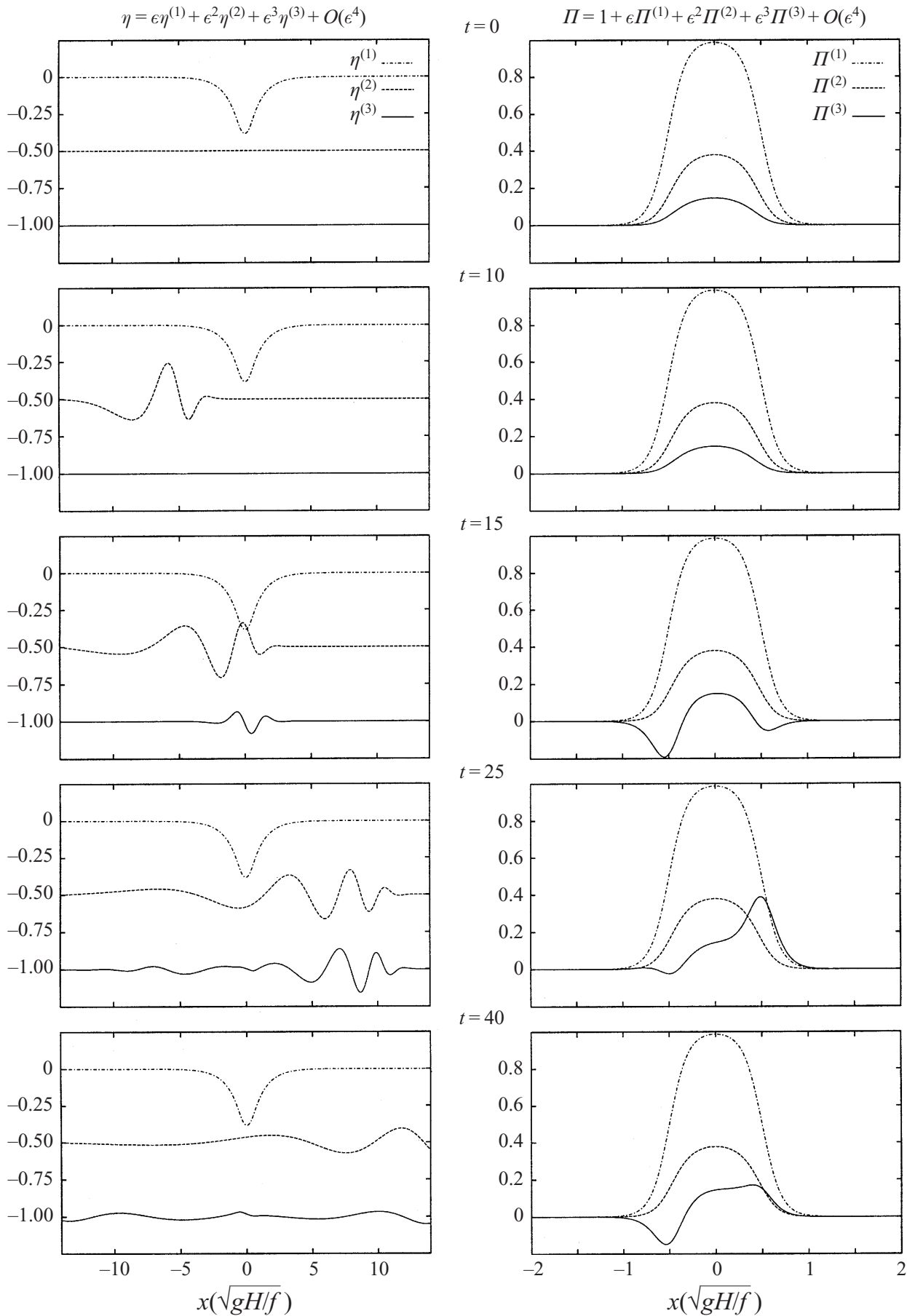

FiguRE 2. Time evolution of the height and potential vorticity asymptotic solutions during a wave-vortex interaction. For the perturbation height plots, the $\eta^{(2)}$ and $\eta^{(3)}$ solutions have been offset from their zero mean values by -0.5 and -1.0 respectively. The vortex parameter $S_{V}=0.2$. $\Pi^{(1)}$ and $\Pi^{(2)}$ are time independent, and plotted for reference only. 
the vortex. We first show, in the asymptotic limit treated in the previous section, that the IG waves leave no trace on the vortex. This is accomplished by showing that in the limit $t \rightarrow \infty$, the time-dependent $O\left(\epsilon^{2}\right)$ and $O\left(\epsilon^{3}\right)$ solutions vanish so that the full solution (up to this order) reduces to the steady $O(\epsilon)$ vortex solution. Second, we show that this long-time non-interaction result must hold to all orders in $\epsilon$. In either case, we need make no assumption on the form of the vortex (other than it being in exact balance), or the initially unbalanced wave-generation region. We conclude this section with a brief discussion clarifying why this surprising no-net-interaction result is not necessarily a consequence of the one-dimensional nature of our problem.

\subsection{The small-amplitude case}

To show that in the limit $t \rightarrow \infty$ the $O\left(\epsilon^{2}\right)$ solutions vanish, it is more convenient to work directly with the equations of motion, rather than taking the limit of the explicit solutions (19)-(21). The only steady bounded solution to (18) is $u^{(2)}=0$. To find $\eta^{(2)}(x, t)$ and $v^{(2)}(x, t)$ in the limit $t \rightarrow \infty$, one may drop the time-derivative and $u^{(2)}$ terms in (16), yielding geostrophic balance at the final state:

$$
\eta_{x}^{(2)}(x, \infty)=v^{(2)}(x, \infty)
$$

This is the familiar geostrophic degeneracy problem (Gill 1982). To close the system, one needs an extra equation. Using (35) and (17) it follows that

$$
v_{x}^{(2)}(x, \infty)-\eta^{(2)}(x, \infty)=v_{x}^{(2)}(x, 0)-\eta^{(2)}(x, 0)=0 .
$$

Now (50) and (51) are used to solve for $v^{(2)}$ and $\eta^{(2)}$ at steady state. Eliminating $v^{(2)}$ yields

$$
\eta_{x x}^{(2)}(x, \infty)-\eta^{(2)}(x, \infty)=0
$$

The requirement that the solution be bounded at $|x| \rightarrow \infty$ again implies that $\eta^{(2)}(x, \infty)=0$ and thus $v^{(2)}(x, \infty)=0$. Hence in the limit $t \rightarrow \infty$ the entire $O\left(\epsilon^{2}\right)$ solution vanishes.

Next, we show that the $O\left(\epsilon^{3}\right)$ fields also vanish in the limit $t \rightarrow \infty$. That $u^{(3)}$ vanishes in that limit is immediately seen from (25) and the fact that $\mathscr{I}_{W V}(x, \infty)=0$. As for $\eta^{(3)}$ and $v^{(3)}$, one has again

$$
\eta_{x}^{(3)}(x, \infty)=v^{(3)}(x, \infty)
$$

Moreover, (23) implies that

$$
\frac{\partial\left(v_{x}^{(3)}-\eta^{(3)}\right)}{\partial t}=-u^{(2)} \frac{\partial^{2} v^{(1)}}{\partial x^{2}}-\frac{\partial u^{(2)}}{\partial x} \frac{\partial v^{(1)}}{\partial x}+\frac{\partial\left(\eta^{(1)} u^{(2)}\right)}{\partial x},
$$

which we integrate with respect to $t$ to obtain

$$
v_{x}^{(3)}(x, t)-\eta^{(3)}(x, t)=P(x, t)
$$

where

$$
\begin{aligned}
P(x, t) & \equiv-\left[\left(v^{(1)}\right)_{x}-\eta^{(1)}\right]_{x} \int_{0}^{t} u^{(2)}(x, s) \mathrm{d} s-\left[\left(v^{(1)}\right)_{x}-\eta^{(1)}\right] \int_{0}^{t} u_{x}^{(2)}(x, s) \mathrm{d} s \\
& =\left[\left(v^{(1)}\right)_{x}-\eta^{(1)}\right]_{x} v^{(2)}(x, t)-\left[\left(v^{(1)}\right)_{x}-\eta^{(1)}\right] \eta^{(2)}(x, t) .
\end{aligned}
$$

In the limit $t \rightarrow \infty, v^{(2)}$ and $\eta^{(2)}$, and therefore $P(x, t)$, vanish. It follows from (53) and (55) that $v^{(3)}=\eta^{(3)}=0$.

The conclusion here is that in the limit $t \rightarrow \infty$, the full solution to $O\left(\epsilon^{4}\right)$ is identical to $\boldsymbol{u}^{(1)}$. In other words, the initially balanced vortex reappears intact at long times 
after the waves have propagated away. This non-trivial result is illustrated in figure 3 , where we plot the long-time continuation of the asymptotic solution shown in figure 2. At $t=1400$ (last row of figure 3 ) the total height and PV fields are indistinguishable from their initial values.

For large (but not infinite) $t$, however, the time-dependent component of the PV, i.e. $\Pi^{(3)}$, is associated with a kink in the $\eta^{(3)}$ solution around $x=0$ (see for instance frame $t=200$ in figure 3 ). This kink is the long-time signature of the wave-vortex interaction. The rate at which this kink disappears is the rate at which the term $P(x, t)$ in (55) goes to zero. Since the $O\left(\epsilon^{2}\right)$ fields vanish as $t^{-1 / 2}$ in the limit $t \rightarrow \infty$ (Gill 1982 ), the signature of wave-vortex interaction disappears at that rate. Note that no such kink appears in $\eta^{(2)}$ at large $t$ since (51), in contrast to (55), has a homogeneous right-hand side.

\subsection{The case of arbitrary amplitude}

This surprising 'no net interaction' result can be extended to the case of arbitrary amplitudes. The proof follows Rossby (1938), who studied the adjustment of a locally unbalanced momentum source. We do likewise except that our initial condition includes, in addition to the unbalanced momentum source, a region of exact balance (the vortex). Following Rossby, we adopt a Lagrangian description of the fluid. Geostrophic balance, together with conservation of $y$-momentum and mass between the initial (subscript ' 0 ') and final (subscript ' $\infty$ ') states imply

$$
\begin{gathered}
\frac{\mathrm{d} \eta_{\infty}}{\mathrm{d} x_{\infty}}=v_{\infty}, \\
v_{\infty}-v_{0}=x_{0}-x_{\infty}, \\
\left(1+\eta_{\infty}\right) \frac{\mathrm{d} x_{\infty}}{\mathrm{d} x_{0}}=1+\eta_{0},
\end{gathered}
$$

where $x_{0}$ and $x_{\infty}$ are, respectively, the initial and final positions of each Lagrangian fluid column. One may eliminate $\eta_{\infty}$ and $v_{\infty}$ from the above to obtain a single equation $\uparrow$ for $x_{\infty}$ :

$$
\left(1+\eta_{0}\right)\left(\frac{\mathrm{d}}{\mathrm{d} x_{\infty}}\right)^{2} x_{0}+\frac{\mathrm{d} \eta_{0}}{\mathrm{~d} x_{\infty}} \frac{\mathrm{d} x_{0}}{\mathrm{~d} x_{\infty}}-x_{0}+x_{\infty}-v_{0}=0 .
$$

This equation could in principle be solved to obtain $x_{\infty}\left(x_{0}\right)$. The crux, however, is that this equation does not contain $u_{0}$. This implies that any initial $x$-momentum has no effect on the final solution. In particular, if $\eta$ and $v$ are initially in geostrophic balance, as they are in our problem, then the solution to (59) is simply $x_{\infty}=x_{0}$, in which case (57) and (58) imply that initial and final values of $\eta$ and $v$ are identical. Thus, although any unbalanced initial $x$-momentum source can be used to generate arbitrary IG waves (which eventually must impinge on the vortex), the IG waves leave no signature on the final state.

That $u_{0}$ does not appear in (59) is a result of the restriction of no $y$ variations: $u_{0}$ displays no direct PV signature. Thus, the objection might be raised as to whether the non-interaction result holds if we take the initial region of imbalance to be associated with a non-trivial potential vorticity. We argue that the answer is still affirmative, but first we make clear a certain facet of the problem.

$\dagger$ Rossby derived this equation for the case $\eta_{0}=0$ (no initial height perturbations) in which case the equation becomes linear. See also Boss \& Thompson (1995) for the axisymmetric top-hat case. 

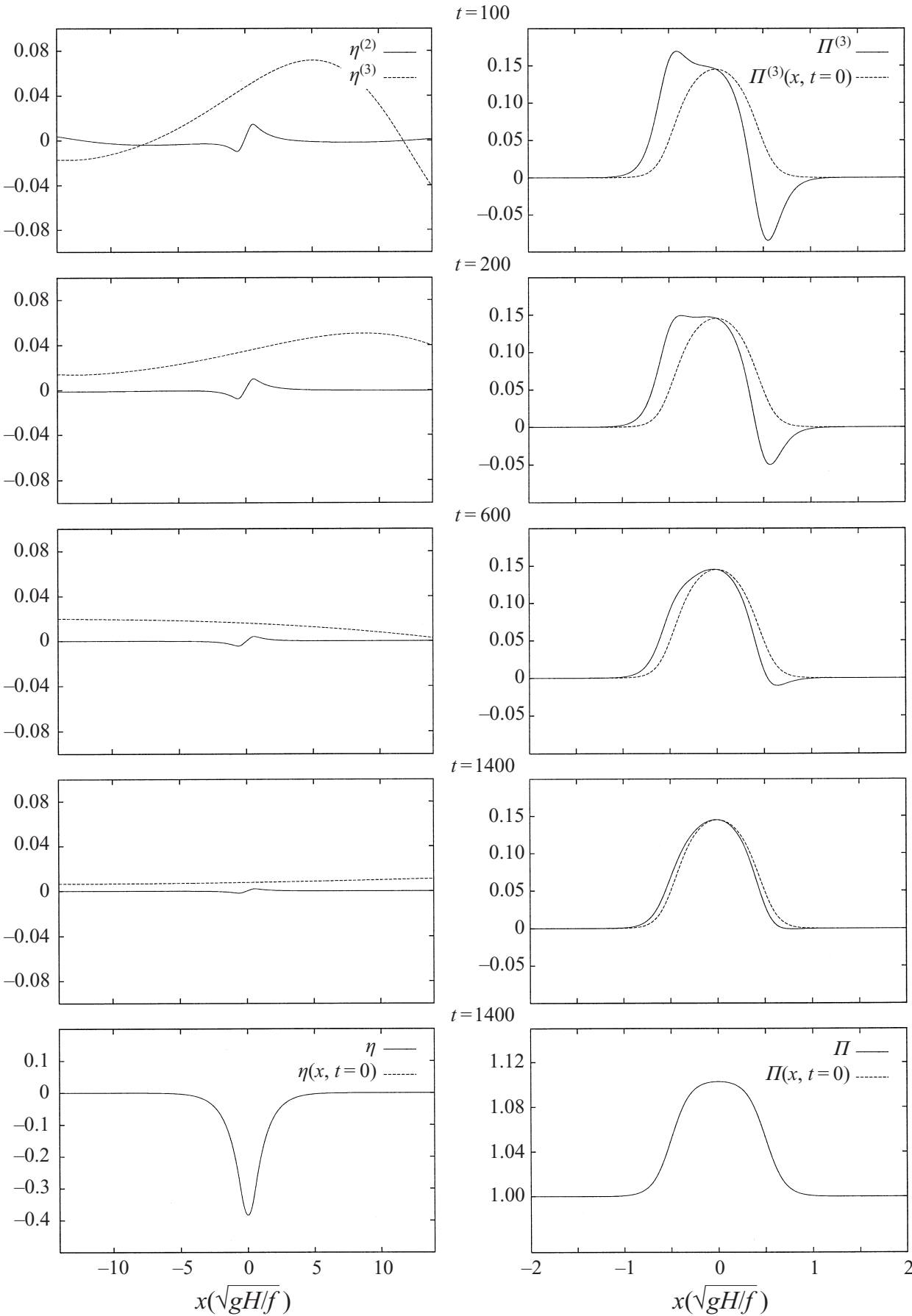

$t=1400$

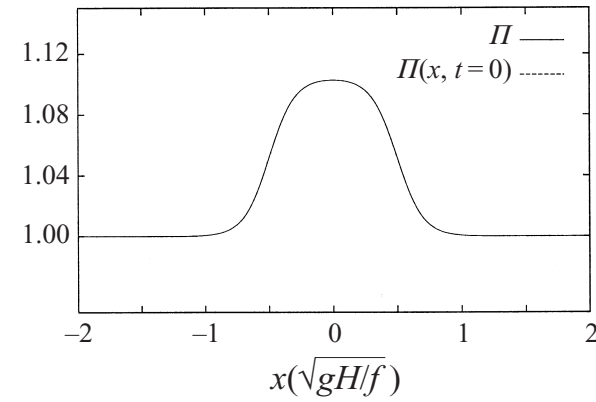

FIGURE 3. Continuation of figure 2 for long times. For the height plots, $\eta^{(0)}$ is no longer plotted and there is no offset as in previous plot. For the potential vorticity plots, we plot only $\Pi^{(3)}(x, t)$ and its initial value, omitting the steady $\Pi^{(0)}, \Pi^{(1)}$ and $\Pi^{(2)}$ fields. The two plots in the last row show the full perturbation solution to $O\left(\epsilon^{4}\right)$ at $t=1400$ along with the initial conditions, where we have chosen $\epsilon=0.1$. 
Assume initial spatial $\eta$ or $v$-velocity variations in the unbalanced region (as opposed to a $u$ variation) which are not exactly balanced. As such, these fields will give rise to IG waves and be associated with non-trivial PV. The PV associated with this local region of imbalance must be physically distinguishable from the PV associated with the localized vortex so that unequivocally one may state this is a problem of an external IG wave interacting with a separate and distinct PV distribution. If such is the case, one may again state that only an exponentially small net long-term wave-vortex interaction will take place. This is because the influence of PV anomalies on the displacement of material particles in the fluid is confined to a few deformation radii (Rossby 1938; Blumen 1972). Specifically, Rossby's original Lagrangian method showed that net material fluid displacements associated with localized unbalanced PV anomalies decrease exponentially with distance from those anomalies. The exponential fall off rate is given by the deformation radius. Thus, the further one is from the IG wave source, the smaller the net interaction.

Note that moving the IG wave source further away from the vortex is not equivalent to requiring the IG waves to be smaller in amplitude. One could take Rossby's (1938) $v$-jet initial conditions for the unbalanced source, simultaneously increase the distance from the balanced region and increase the amplitude of the $v$-jet to obtain IG waves of roughly constant amplitude at a fixed distance from the wave source region.

Finally, note that implicit in the arguments presented in this section is that flows in a one-dimensional infinite domain actually become steady. We have no formal proof of this. However, numerical integrations of the full shallow-water equations (as shown in §5) support this assumption: we have never observed perpetual generation of gravity waves at the expense of the energy of the vortex. In addition, the asymptotic results presented in $\S 3.1$ are consistent with the claim that the oscillatory gravity wave solutions decay in time, and the flow eventually becomes steady.

\subsection{Discussion}

We do not believe that this long-time result is obvious. Consider: why should arbitrarily large IG waves not be able to alter the potential vorticity of the flow, e.g. through Stokes drift? We have shown in the small-amplitude limit, and will show numerically for the case of large amplitudes, that the PV does change in time when the IG waves hit the vortex. However, the net long-time change always turns out to be zero.

At this point, one may be tempted to dismiss the one-dimensional shallow-water equations as too simple (and thus constrained) a model to capture any net wavevortex interactions. It is therefore worth noting that the no-net-interaction result in this problem is not an immediate consequence of one-dimensionality, since it applies only to the case of external IG waves impinging on a balanced vortex. In more general circumstances, a vortex would not be exactly balanced. Hence, not only might a vortex encounter external IG waves, as in this study; it would also generate them while undergoing geostrophic adjustment. Net long-term changes in PV are possible during a pure geostrophic adjustment (i.e. in the absence of external IG waves), even in the simplest one-dimensional context. These changes must be in part due to IG waves because at the level of the quasi-geostrophic approximation in one dimension (where there are no IG waves) the PV cannot be changed. PV changes that persist after the IG waves have propagated away, termed 'irreversible' by Buhler \& McIntyre (1998), occur in a simple adjustment problem, as is evident from the nonlinearity of equation (59), which ultimately determines the final PV configuration given the initial one. We illustrate this with a simple example. 


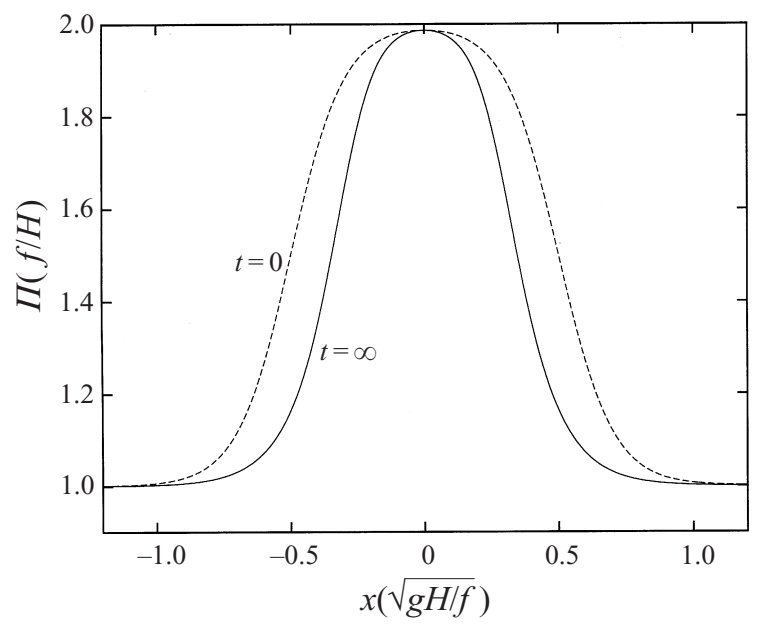

FIGURE 4. Irreversible (after IG waves have propagated away) change in potential vorticity in the one-dimensional shallow-water system, via geostrophic adjustment. The vortex is initially unbalanced, with the vortex parameters initially $A_{V}=1.0$ and $S_{V}=0.2$.

Consider solving (1) with the following initial conditions:

$$
\boldsymbol{u}(x, t=0) \equiv\left(\begin{array}{l}
u \\
v \\
\eta
\end{array}\right)=\left(\begin{array}{c}
0 \\
0 \\
\frac{1}{\Pi(x)}-1
\end{array}\right)
$$

with $\Pi$ given by (61) of $\S 5$. This corresponds to an initial bump of PV and is illustrated in figure 4 (dotted line). Because $v(x, t=0)=0$, the initial conditions are unbalanced; IG waves will be generated as the flow adjusts to geostrophic balance. The final-state PV profile obtained by solving (59) numerically is shown in figure 4 (solid line) where it is seen that the final PV differs quite substantially from the initial one. A detailed discussion of this effect can be found in Kuo \& Polvani (1999).

The point here is that net long-time PV changes can be captured by the onedimensional shallow-water equations, provided an unbalanced IG component of the flow is present. Somehow, however, in the case of external IG waves impinging on a balanced vortex, the net long-time PV change vanishes.

\section{Numerical experiments}

We now present the results of numerical experiments: the aim here is to determine whether the key results of the asymptotic analysis carry over to regimes where amplitudes are not small. We accomplish this by demonstrating, from the full shallowwater equations, the existence of time-dependent wave-vortex interactions, the effect of the IG waves on the vortex, and the curious result of long-time non-interaction.

The numerical experiments consist of integrating (1) in time with initial conditions of the form (5). The initially balanced vortex $\boldsymbol{u}_{V}$ is specified by inverting the following potential vorticity profile:

$$
\Pi(x, t=0)=1+\frac{A_{V}}{2}\left(\tanh \left[\frac{\left(x+\frac{1}{2}\right)}{S_{V}}\right]+\tanh \left[\frac{\left(-x+\frac{1}{2}\right)}{S_{V}}\right]\right),
$$


while the initial unbalanced field $\boldsymbol{u}_{W}$ is specified by

$$
u_{W}=A_{W} \mathrm{e}^{-\left(x-x_{0}\right)^{2}}
$$

with $x_{0}=-14$ (as in $\S 3.4$ ). The three parameters appearing in the problem are thus $A_{V}, S_{V}$, and $A_{W}$, representing respectively the strength and edge steepness of the initial vortex and the amplitude of the incoming IG waves. These initial conditions are shown schematically in figure 1.

The full shallow-water equations are numerically integrated with a high-order accurate WENO (weighted essentially non-oscillatory) scheme. This method was originally developed in the context of gas dynamics to handle fluid flow discontinuities (i.e. shocks and bores). Because of the potentially high Rossby/Froude numbers involved in these numerical experiments, bores are likely to form, even in the presence of rotation (Houghton 1969). A numerical scheme designed to capture these features of the flow, without excessive dissipative or dispersive effects, is therefore necessary. The WENO scheme has been tested extensively (Jiang \& Shu 1996). It is a finite difference scheme based on maximizing the weighting coefficients of a moving stencil to achieve fifth-order accuracy in space, while employing a Runge-Kutta scheme to obtain fourth-order accuracy in time. Near discontinuities the spatial accuracy of the code decreases to third order. The code represents an improvement, in terms of accuracy and vectorizability, over the older, better-known ENO (essentially nonoscillatory) scheme (Liu, Osher \& Chan 1994) and can achieve up to 750 MFlops on a Cray T-90.

For all of the numerical experiments shown here, we use a resolution of 40 or more grid points per deformation radius. At the boundaries of the computational domain we use Sommerfeld outflow conditions (Sommerfeld 1964). Furthermore, the size of the computational domain is chosen to be orders of magnitude larger than the wave-vortex interaction region, so that any IG waves potentially reflected from the boundaries would have no effect on the vortex. Specifically, in the long-time integrations we use a domain 320 deformation radii wide.

In order to ascertain whether the passage of the IG waves leaves a net, long-time effect on the vortex, it is necessary to damp the generation of IG waves in the initially unbalanced region. This is because the adjustment process which produces the IG waves near $x=x_{0}$ would otherwise take prohibitively long to complete, typically several hundred inertial periods (Kuo \& Polvani 1997). We have chosen to implement the damping by adding the term $\frac{1}{2} u(x, t) X(x) T(t)$ to the right-hand side of $x$-momentum equation in $(1)$, where $X(x) \equiv-(\tanh (x+16)+\tanh (-x-25))$ and $T(t) \equiv \tanh \left(t-t_{0}\right)$. This confines the damping to the wave generation region only, and starts acting after $t \approx t_{0}=10$. It should be clear that our results regarding the existence and nature of wave--vortex interactions are independent of this damping, since it only acts in a region far away from where the interaction takes place. Any modifications to the $\mathrm{PV}$ in the damping region are too far to be advected into the vortex region. This fact has been verified by examining the PV field throughout the computational domain during the integrations.

\subsection{Interaction dependence on the $I G$ wave amplitude}

We start by exploring how the interaction depends on the amplitude $A_{W}$ of the IG waves. A first example is shown in figure 5, where the evolution of the height (left column) and PV (right column) fields is shown for the case $A_{W}=1.4$. Notice that the left column spans a region 20 deformation radii wide, whereas the right column 

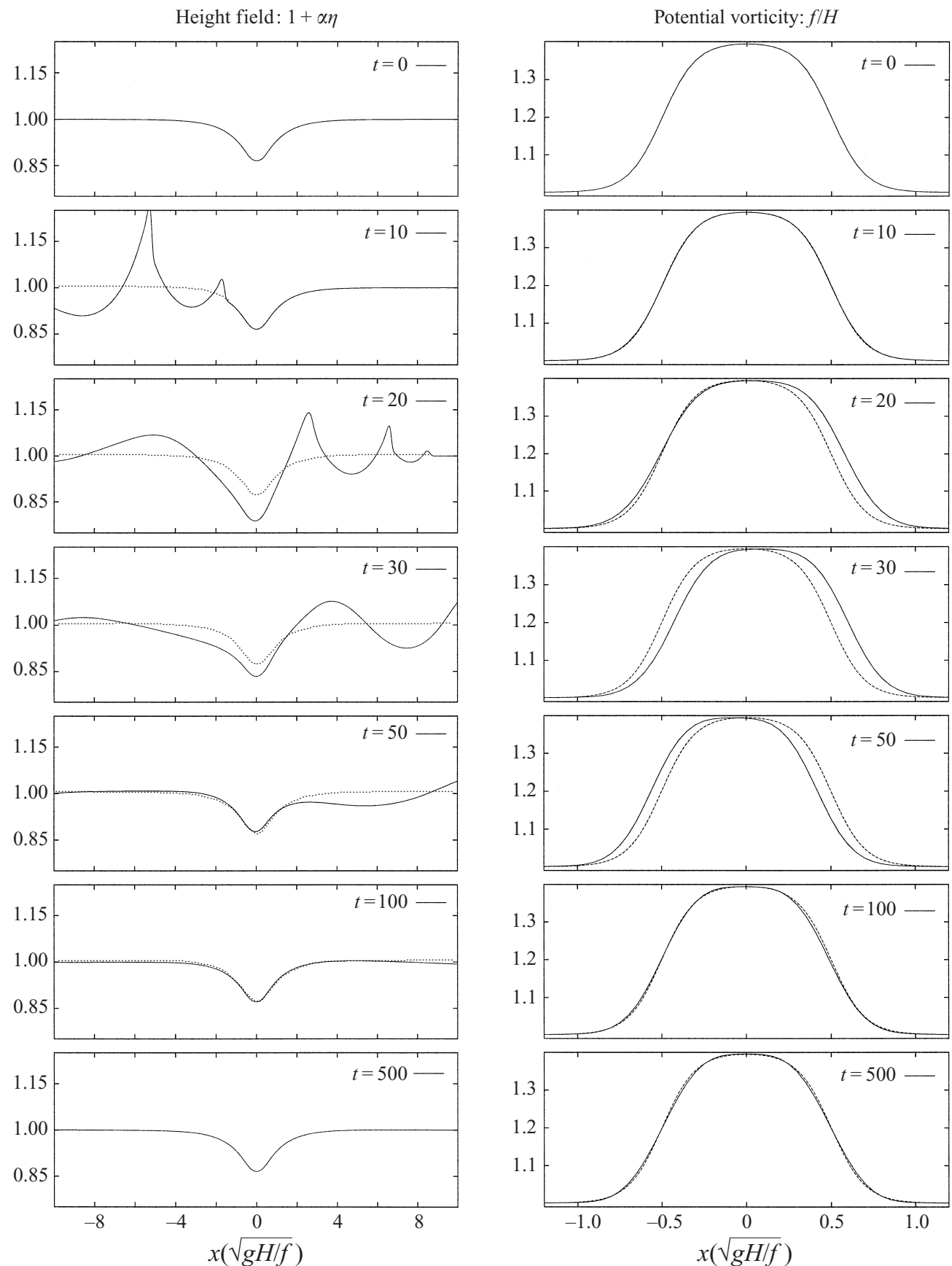

FiguRE 5. Height and potential vorticity fields plotted at selected times from $t=0$ (dotted lines except in top row) to $t=500$ for the case $A_{W}=1.4$. The vortex parameters are $A_{V}=0.4$ and $S_{V}=0.2$

is focused on a much smaller region approximately 2 deformation radii wide, centred on the vortex.

At $t=10 \mathrm{IG}$ waves propagate in from the left and are just about to hit the vortex. At this time, the PV is unaffected. In the next two frames, as the waves pass through 
the vortex, the PV is noticeably altered. The PV here is not merely advected uniformly as a whole: a very clear expansion can be seen at $t=20$. As time progresses, the IG waves decay in amplitude and their effects on the PV are diminished. Finally, at long times $(t=500)$, both the height and PV fields recover their original shape, in agreement with the no-net-interaction result discussed in the previous section.

As the wave amplitude $A_{W}$ is increased, the interaction becomes stronger. In figure 6 , the case $A_{W}=3.2$ is illustrated. The larger wave amplitude is reflected in the scale change for the height field plots. The stronger interaction is apparent in the PV plots (cf. $t=20$ and $t=30$ in particular). At $t=500$, the PV has not quite recovered its initial shape (the difference is slightly greater than for the previous case $A_{W}=1.4$ ); this is simply due to the higher IG wave activity at this time. It is quite remarkable that, even with large bores hitting the vortex (cf. the height field at $t=10$ ), the vortex eventually emerges intact: the predicted result of an unchanged final state is very nearly realized.

We next present some diagnostic summaries of the preceding computations. Figure $7(a-c)$ shows, as functions of time, the RMS averages of the field variables $u, v$ and $\eta$. These quantities were computed in the region $x \in[-6,6]$ where wave-vortex interaction occurs. The RMS fields do not change until $t \approx 10$, at which point the IG waves enter the region of interest. Then a sharp rise occurs, the maximum change occurring around $t \approx 40$, after which the RMS fields slowly recover and approach their initial values. For both values of $A_{W}$, the trends suggest no net changes at long times.

Alternatively, one can diagnose the interaction in terms of the energy variation in the vortex region. In figure $7(d)$ we have plotted, versus time, the total energy $E$ defined in (41) in the same region. The figure shows the energy initially increasing due to the incoming IG waves and subsequently decaying back to its initial value (as the IG waves decrease in amplitude), implying no net energy exchange between the IG waves and the vortex.

Finally, we describe in more detail how the vortex is affected by the IG waves by examining the time evolution of the vortex energy and PV. At each time $t$, the balanced fields $v_{b}(t)$ and $\eta_{b}(t)$ can be constructed by exact inversion of the instantaneous PV, as described in $\S 2$. The balanced energy is then computed using the expression $E_{b} \equiv \int\left[\frac{1}{2} v_{b}^{2}\left(1+\eta_{b}\right)+\frac{1}{2}\left(1+\eta_{b}\right)^{2}\right] \mathrm{d} x$, where the integration is carried out over the interaction region $x \in[-6,6] . E_{b}$ can be thought of as the energy of the flow if the flow were instantaneously exactly balanced by setting the unbalanced components of the flow instantaneously to zero.

The balanced energy $E_{b}$, normalized by its initial value is plotted in figure $8(a)$. As expected, $E_{b}$ is unaffected for approximately $t<10$, at which point the IG waves begin to enter the vortex region. As the IG waves interact with the vortex, $E_{b}$ varies, a clear indication that energy is exchanged between the balanced and unbalanced components of the flow. The two cases of $A_{W}$ show, as might be expected, that larger IG waves lead to stronger interactions. At long times, $E_{b} \rightarrow 1$ for both values of $A_{W}$; that is, there is no net gain or loss to $E_{b}$ over very long time scales.

Finally, we look more closely at the PV. We plot the RMS of $[P V(x, t)-P V(x, 0)]$, the deviation of the potential vorticity from its initial value in figure $8(b)$. We chose a smaller RMS averaging region in the case, $x \in[-3,3]$, because the PV associated with the vortex is only about one deformation radius wide. Figure 8 again demonstrates that the vortex is severely affected by the IG waves and that larger wave amplitude leads to stronger interaction. Towards the end of the integration, the IG wave effects disappear. 

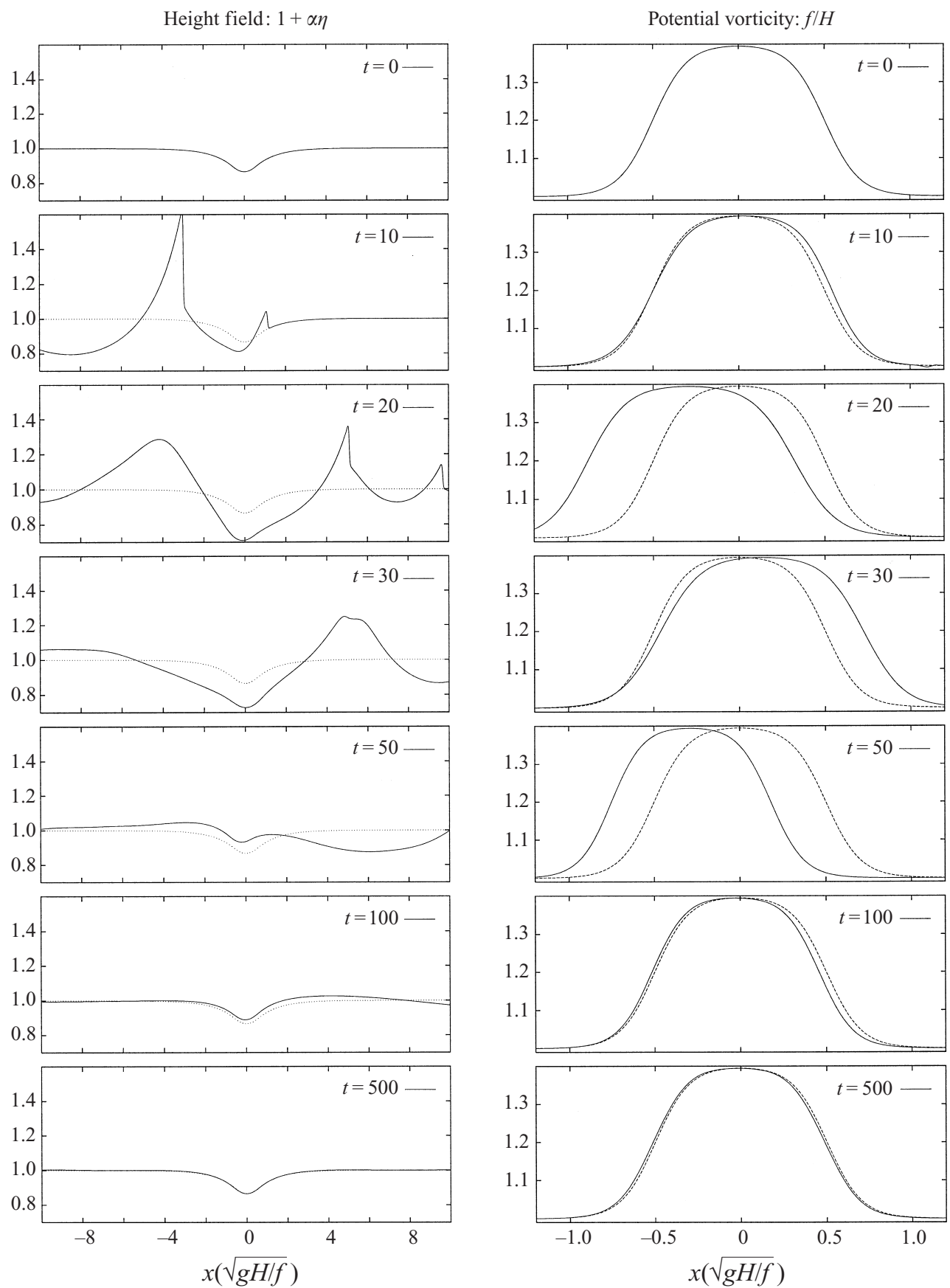

FIGURE 6. As in figure 5, but for a large wave amplitude $A_{W}=3.2$. The wave-vortex interaction is stronger in this case.

\subsection{Interaction dependence on the vortex parameters}

In this subsection we explore how the wave-vortex interaction depends on the vortex amplitude $A_{V}$ and vortex edge steepness $S_{V}$. Moreover, we are interested in how the interaction might depend on the cyclonicity of the vortex. 

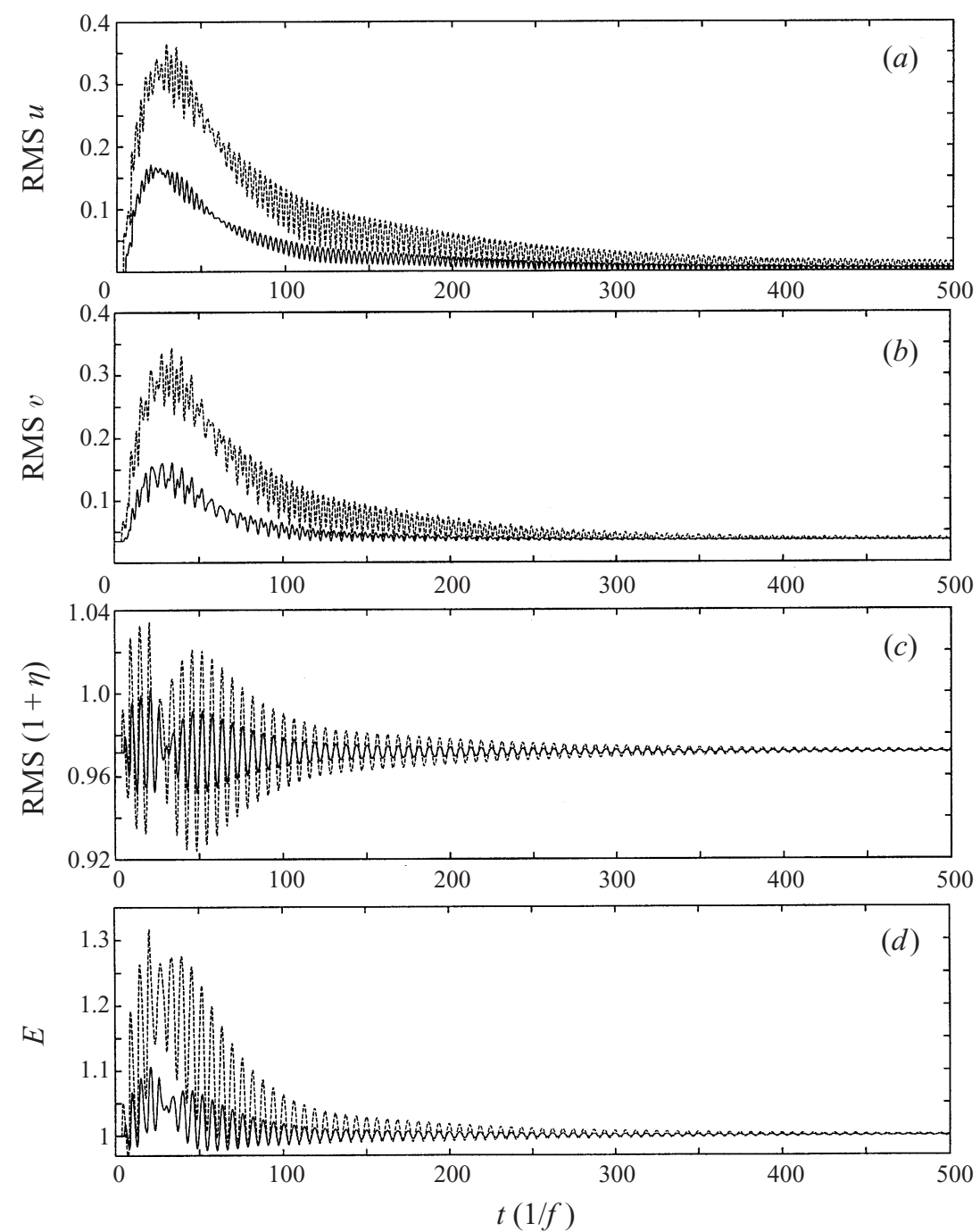

FiguRE 7. Root mean square values of $(a)$ the $u,(b) v$, and $(c)$ height fields as well as $(d)$ the energy (normalized to one initially) in the region $x \in[-6,6] . A_{W}=1.4$ (solid line) and 3.2 (dashed line), as for figure 5 and 6 .

Six different initial vortices are illustrated in figure 9. Three are cyclones $(\mathrm{PV}>1)$ and three are anticyclones $(\mathrm{PV}<1)$. Each cyclone can be paired with a corresponding anticyclone of similar amplitude; the values of $A_{V}$ in (61) are chosen in such a way that $v$ and $\eta$ are very nearly mirror images of one another for each pair. The parameter $S_{V}=0.2$ was used in all six cases.

To measure the time-dependent interaction, we compute the area under the PV curve

$$
a(t) \equiv \frac{1}{N} \int_{-3}^{3}|\Pi(x, t)-1| \mathrm{d} x,
$$

where the normalization factor $N=\int_{-3}^{3}[\Pi(x, 0)-1] \mathrm{d} x$ is the area under the initial 

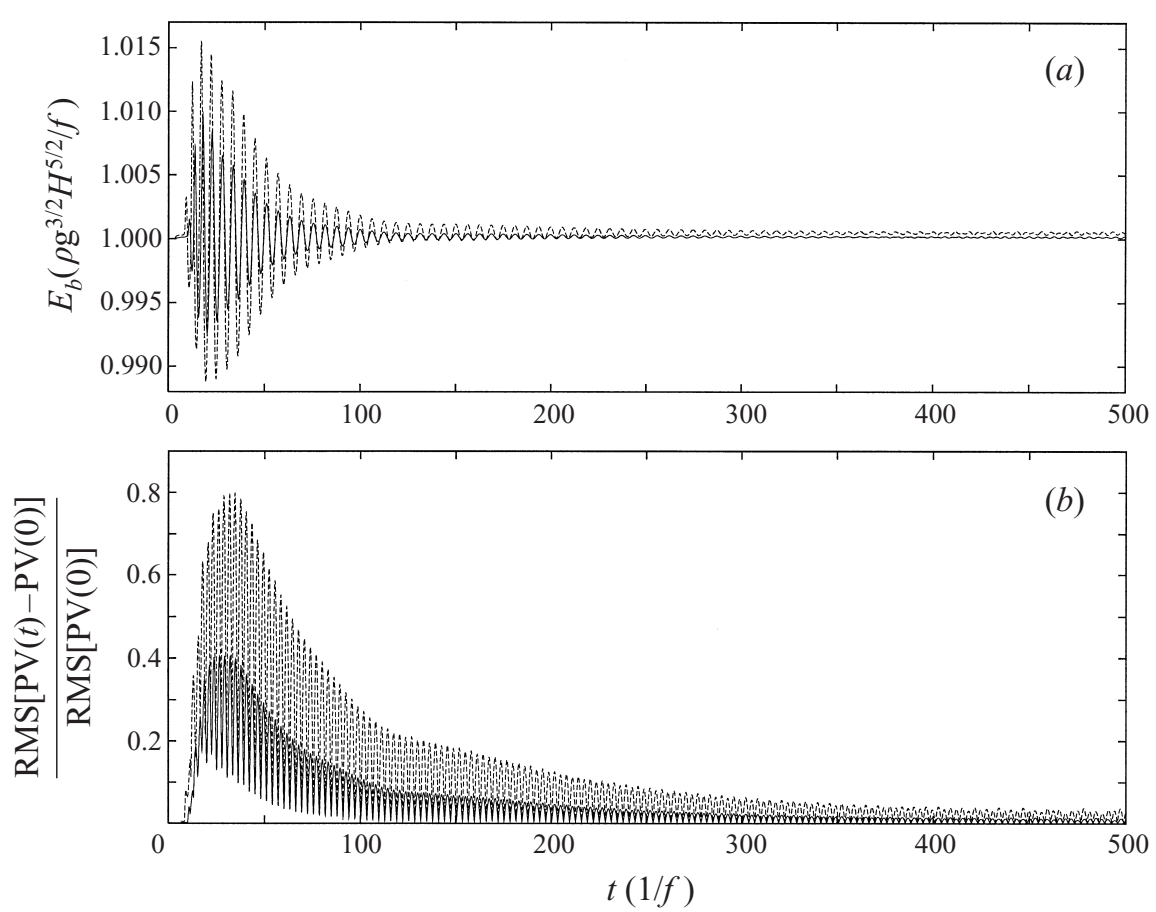

FiguRE 8 . The effect of IG waves on the vortex. (a) The balanced energy $E_{b}$ (normalized by its initial value), which is the energy associated with the vortex. (b) The RMS difference in potential vorticity from initial values, also normalized. Larger wave amplitudes (larger $A_{W}$ ) leads to greater distortion of the vortex. $A_{W}=1.4$ (solid line) and $A_{W}=3.2$ (dashed line).

vortex after subtracting the mean 1 . We have computed several other measures that describe the interaction, but we present this particular one because it brings out the key points in the clearest way. For an identical wave amplitude $A_{W}=2.0$, we have plotted in figure 10 the evolution of $a(t)$ for all six vortices shown in figure 9 . Since results are plotted for relatively short times, no damping in the wave generation region was necessary.

A first result from this set of numerical experiments is that the magnitude of relative PV changes can be substantial. For the initial conditions of figure 9, the area under the PV curve can vary up to $30 \%$, as illustrated in figure 10 . Of course this number will depends on $A_{W}$ as well. It should be noted that, for the cases shown in figure 9, the advective Rossby number is not at all extreme: based on the RMS $u$-velocity in the region $x \in[-6,6]$, the maximum Rossby number is only about 0.25 .

A second result is that there is a marked difference between the cyclonic vortices (figure 10a) and the anticyclonic ones (figure 10b). Cyclones appear to interact more strongly with IG waves than do anticyclones, at least in terms of relative PV changes. It was shown in Polvani et al. (1994) that in freely evolving two-dimensional shallowwater turbulence, cyclonic vortices are less coherent and less axisymmetric than anticyclonic vortices and that the IG wave generation regions are highly correlated with the locations of cyclonic vortices. If our result holds in two dimensions, it might provide additional explanation for the weaker coherence of cyclonic vortices compared to their anticyclonic counterparts.

A third result to be gathered from figure 10 is that the wave-vortex interaction is not strongly dependent on the vortex amplitude. For instance, quadrupling $A_{V}$ from 

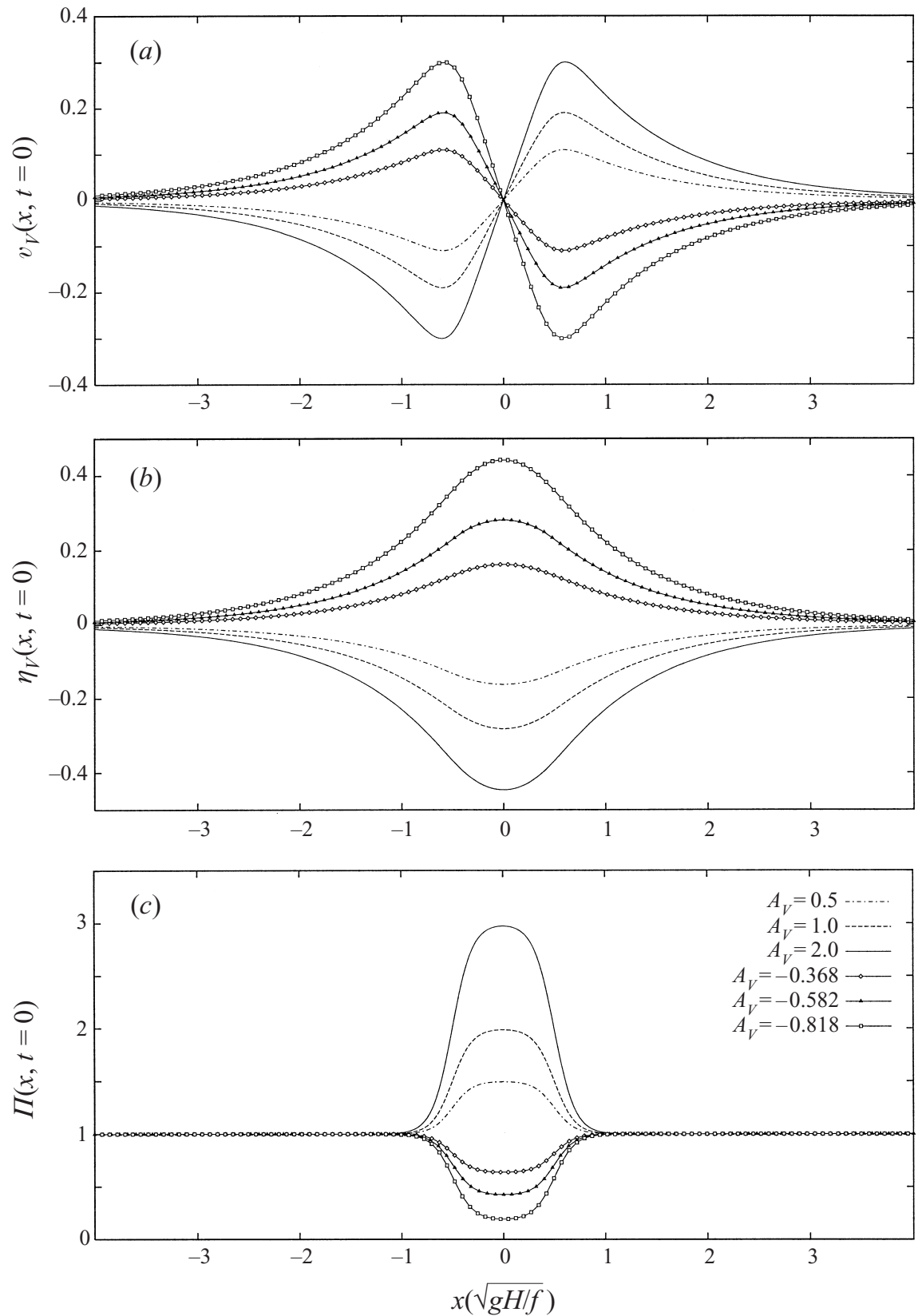

Figure 9. The six initial conditions used to study the interaction dependence on the vortex amplitude $A_{V}$. Positive (negative) values of $A_{V}$ correspond to cyclonic (anticyclonic) vorticity in the strip $x \in[-1,1]$. (a) $v_{V},(b)$ the corresponding balanced $\eta_{V}$, where cyclones (anticyclones) correspond to surface depressions (elevations) and (c) the PV. For all these cases $S_{V}=0.2$.

0.5 to 2.0 only leads to roughly an $8 \%$ increase in $a(t)$. Furthermore, in the cyclonic case the interaction increases with vortex amplitude, whereas the opposite is true in the anticyclonic case. Again, if these results generalize to two-dimensional vortices, they might help explain the findings of Polvani et al. (1994). In those turbulence simulations, as initially small vortices grow in size through repeated mergers, those 

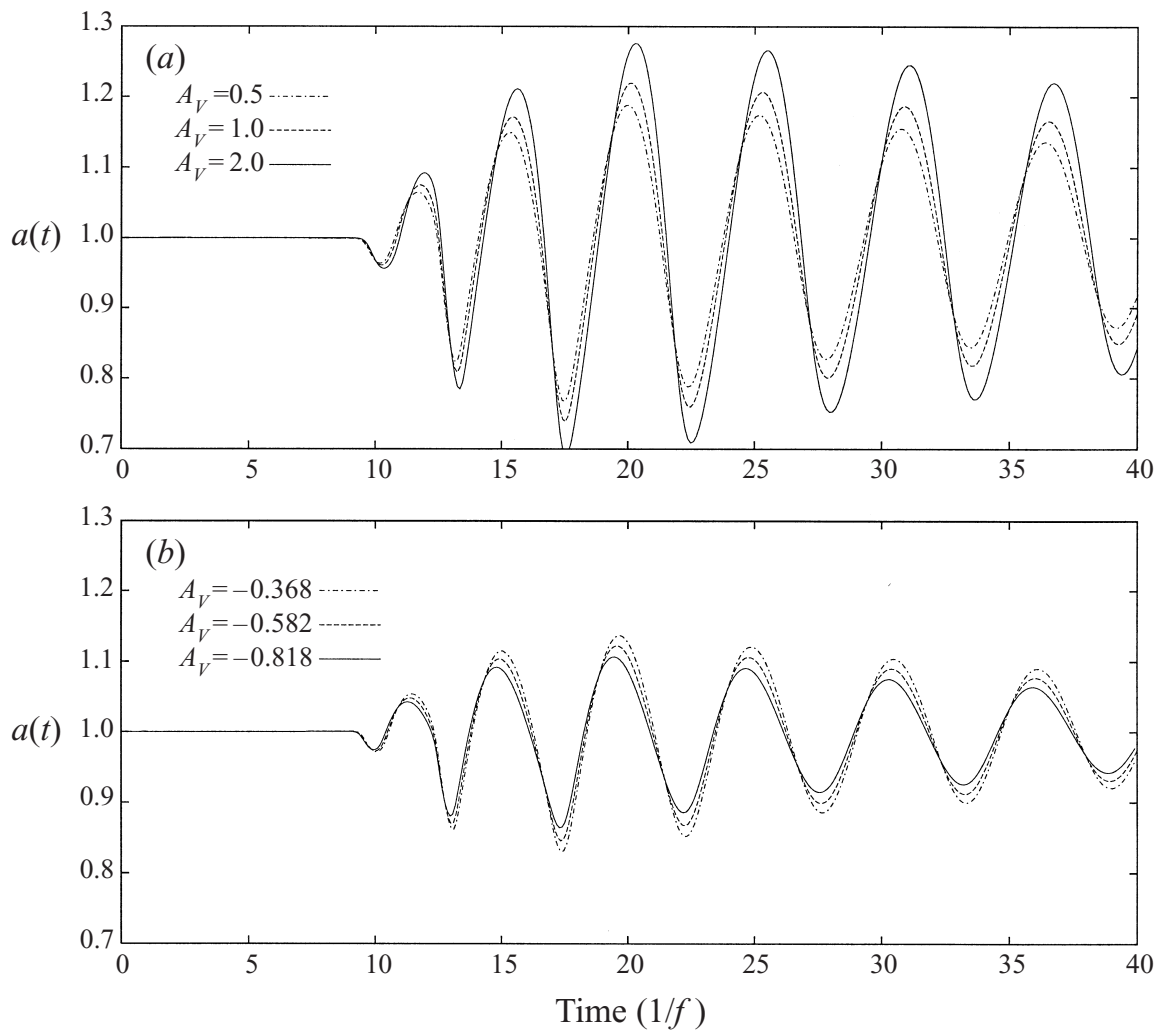

FIgURE 10. The time evolution of $a(t)$, the area under the PV anomaly, for $(a)$ the cyclonic vortices and $(b)$ the anticyclonic vortices. The initial conditions were shown in figure 9. The IG waves hit the vortex at $t \approx 10$, at which time the PV is altered. For all these cases $A_{W}=2.0$ and $S_{V}=0.2$.

vortices which interact more strongly with IG waves would presumably be less likely to remain coherent and axisymmetric, as in the case of cyclones.

Finally, we mention that the steepness parameter $S_{V}$ has little effect on the wavevortex interaction. Figure 11 shows that increasing the vortex edge steepness leads only to slightly greater changes in $a(t)$ when the maximum amplitude of the vortex is held fixed.

\section{Discussion}

In this paper, we have demonstrated the existence of wave-vortex interaction in rotating shallow water by considering how, in physical space, a train of IG waves is able to modify a vortex. The strength of this formulation (as opposed to the Fourier space formulation) lies in the fact that the results obtained do not depend on either small amplitude (near linear) or statistically homogeneous assumptions, and that it allows us to describe when, where and how the interaction occurs. While it is well known that balanced motions can affect IG waves, we have here shown explicitly that IG waves can affect balanced motions as well. For small amplitudes, the interaction scales as the square of the Rossby number. For larger amplitude (e.g. maximum advective Rossby numbers of no more than 0.3 ) the interaction results in substantial temporal changes in PV (up to 30\%). Moreover, it was found that IG waves induce greater PV changes on cyclonic vortices than on anticyclonic vortices. Finally, we 

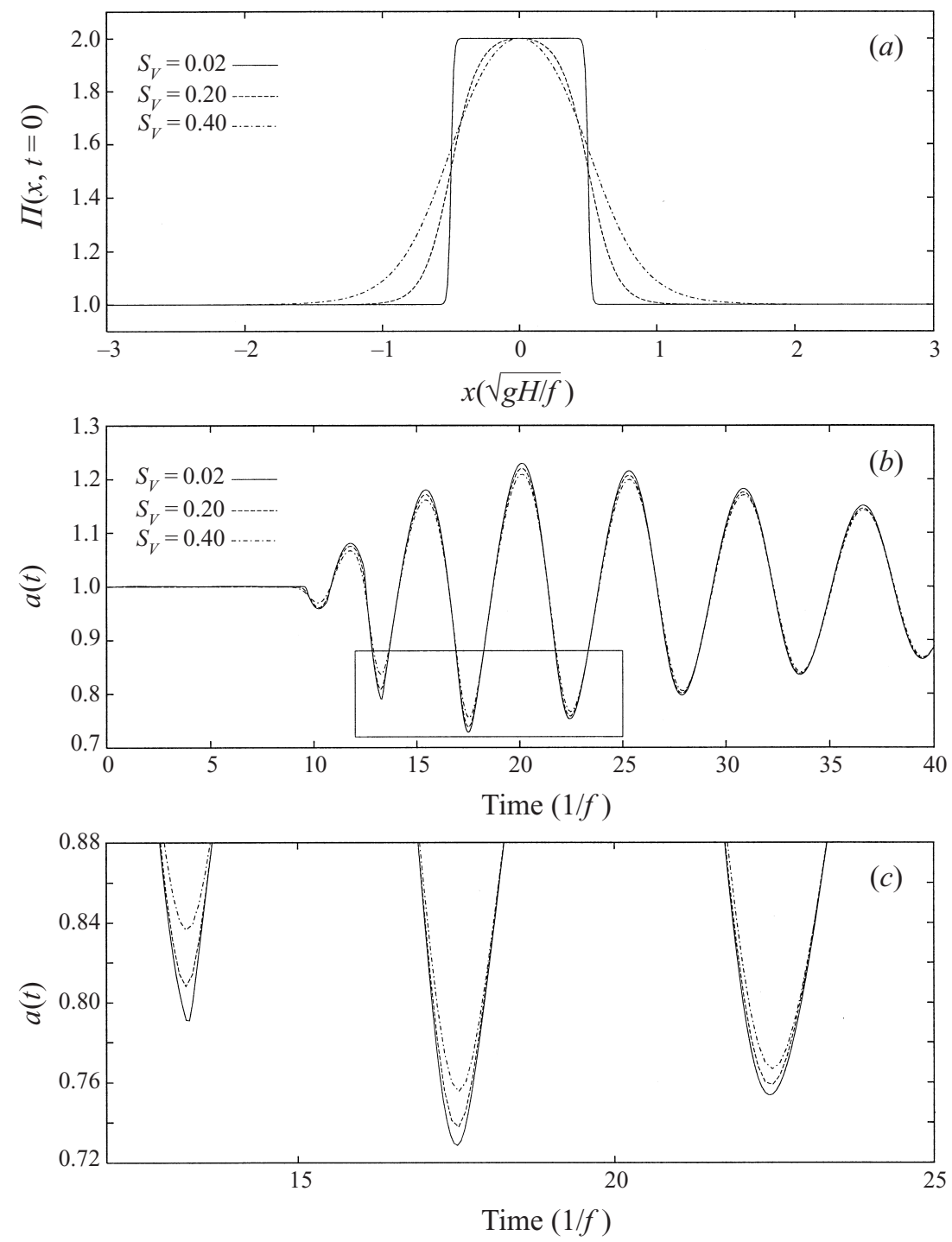

FIGURE 11. The interaction dependence on $S_{V}$, the vortex steepness parameter. $(a)$ The initial PV profiles, $(b) a(t)$ vs. time, $(c)$ is an enlargement of the boxed region in $(b)$. In all cases, the dependence on $S_{V}$ is slight. The IG wave parameter was fixed at $A_{W}=2.0$ and the vortex amplitude $A_{V}=2.0$.

have demonstrated that, surprisingly, in the simple one-dimensional context we have studied here, IG waves leave no trace on the vortices in the limit $t \rightarrow \infty$, irrespective of the wave amplitude.

From this infinite-time result, however, one should not conclude that there is no wave-vortex interaction, and that therefore gravity waves 'don't matter' (cf. Buhler \& McIntyre 1998). For instance, the instantaneous temporal changes in PV induced by IG waves have important implications for the validity of balanced models. The diagnostic step needed in any balanced model relies crucially on inverting the instantaneous PV field for calculating balanced velocity components (which are then used to advect the PV). As we have shown, IG waves do affect the PV field on time scales greater than $1 / f$; hence, they do matter. In the simplest balanced model, 
quasi-geostrophy, PV cannot change in time in a one-dimensional setting. Therefore, the extent to which the PV changes is the extent to which the quasi-geostrophic theory fails, in this one-dimensional model at least.

Ideally, one would like to be able to accurately predict slow balanced motions in the presence of gravity waves without using the full primitive equations. Such a problem has been the subject of intense research (Charney 1951; Lorenz 1960; Gent \& McWilliams 1983; Salmon 1985; McWilliams 1985; Williams 1985; Allen, Barth \& Newberger 1990; Spall \& McWilliams 1992; Vallis 1992; McIntyre \& Norton 1990, among many). The work presented here does not address this fundamental prognostic problem but at least shows clearly how balanced motion can be altered by gravity waves. Hopefully, it might suggest new insights into the construction of balanced models.

A main reason for our first presenting results using a simplified one-dimensional model, as opposed to working in a more realistic two-dimensional setting, is that the general features of wave-vortex interaction can be elucidated in simplest form in this case, with the advantage of having exact PV inversion diagnostics. There is every reason to expect that the time-dependent changes in PV found here will also occur in two dimensions (in addition, of course, to the even richer dynamical possibilities associated with the added dimension). An investigation into the interaction problem in two dimensions is forthcoming.

We would like to thank Guang Shan Jiang and Chi Wang Shu for providing us with the Euler equation version of their WENO code. The authors also benefited from discussions with Rupert Ford, Joseph Keller, and Oliver Buhler, and a wealth of useful suggestions on the manuscript from an anonymous reviewer. Numerical calculations were performed on the Pittsburgh Supercomputing Center's CRAY C-90 and the San Diego Supercomputer Center's Cray T-90. Funding was provided by the National Science Foundation.

\section{REFERENCES}

Allen, J. S., Barth, J. A. \& Newberger, P. A. 1990 On intermediate models for barotropic continental shelf and slope flow fields. Part I: Formulation and comparison of exact solutions. J. Phys. Oceanogr. 20, 1017-1042.

BARTEllo, P. 1995 Geostrophic adjustment and inverse cascades in rotating stratified turbulence. $J$. Atmos. Sci. 52, 4410-4428.

Blumen, W. 1972 Geostrophic adjustment. Rev. Geophys. Space Phys. 10, 485-528.

Boss, E. \& Thompson, L. 1995 Energetics of nonlinear geostrophic adjustment. J. Phys. Oceanogr. 25, 1521-1529.

BuHLER, O. \& MCINTYRe, M. 1998 On non-dissipative wave-mean interactions in the atmosphere or oceans. J. Fluid Mech. 354, 301-343.

CAHn, A. 1945 An investigation of the free oscillations of a simple current system. J. Met. 2, $113-119$.

Charney, J. G. 1951 Dynamic forecasting by numerical process. In Compendium of Meteorology. American Meteorological Society, 1334 pp.

Dewar, W. K. \& KillworTh, P. D. 1995 Do fast gravity waves interact with geostrophic motions? Deep-Sea Res. I 42, 1063-1081.

ERrico, R. M. 1984 The statistical equilibrium solution of a primitive equation model. Tellus 36A, 42-51.

FARGE, M. \& SADOURnY, R. 1989 Wave-vortex dynamics in rotating shallow water. J. Fluid Mech. 206, 433-462.

FORD, R. 1994 Gravity wave radiation from vortex trains in rotating shallow water. J. Fluid Mech. 281, 81-118. 
Gent, P. R. \& McWilliams, J. C. 1983 Consistent balanced models in bounded and periodic domains. Dyn. Atmos. Oceans 7, 67-93.

GiLl, A. E. 1976 Adjustment under gravity in a rotating channel. J. Fluid Mech. 103, 275-295.

GiLl, A. E. 1982 Atmosphere-Ocean Dynamics. Academic, 662 pp.

Houghton, D. D. 1969 Effect of rotation on the formation of hydraulic jumps. J. Geophys. Res. 74, 1351-1360.

JiAng, G. S. \& SHU, C. W. 1996 Efficient implementation of weighted ENO schemes. J. Comput. Phys. 126, 202-228.

JoHn, F. 1978 Partial Differential Equations. Springer, 198 pp.

Kevorkian, J. \& Cole, J. D. 1981 Perturbation Methods in Applied Mathematics. Springer, 558 pp.

Kuo, A. C. \& Polvani, L. M. 1997 Time-dependent fully nonlinear geostrophic adjustment. J. Phys. Oceanogr. 27, 1614-1634.

Kuo, A. C. \& Polvani, L. M. 1999 Geostrophic adjustment, cyclone/anticyclone asymmetry, and potential vorticity rearrangement. Phys. Fluids. To be submitted.

LELONG, M. P. \& Riley, J. J. 1991 Internal wave-vortical mode interactions in strongly stratified flows. J. Fluid Mech. 232, 1-19.

LeVeque, R. J. 1992 Numerical Methods for Conservation Laws. Birkhauser, 214 pp.

LiU, X. D., Osher, S. \& Chan, T. 1994 Weighted essentially non-oscillatory schemes. J. Comput. Phys. 115, 200-212.

LoRENZ, E. N. 1960 Energy and numerical weather prediction. Tellus 12, 364-373.

Majda, A. J. \& EmbID, P. 1998 Averaging over fast gravity waves for geophysical flows with unbalanced initial data. Theor. Comput. Fluid Dyn. 11, 155-169.

McIntyre, M. E. \& Norton, W. A. 1990 Dissipative wave-mean interactions and the transport of vorticity or potential vorticity. J. Fluid Mech. 212, 403-435.

McWilliams, J. C. 1984 The emergence of isolated coherent vortices in turbulent flow. J. Fluid Mech. 146, 21-43.

McWilliams, J. C. 1985 A uniformly valid model spanning the regimes of geostrophic and isotropic, stratified turbulence: balanced turbulence. J. Atmos. Sci. 42, 1773-1774.

Pedlosky, J. 1982 Geophysical Fluid Dynamics, Chap. 3. Springer.

Polvani, L. M., McWilliams, J. C., Spall, M. A. \& Ford, R. 1994 The coherent structures of shallow water turbulence: deformation radius effects, cyclone/anticyclone asymmetry and gravity wave generation. Chaos 4, 177-186.

RossBy, C. G. 1938 On the mutual adjustment of pressure and velocity distributions in certain simple current systems II. J. Mar. Res. 1, 239-263.

SAlmon, R. 1985 New equations for nearly geostrophic flow. J. Fluid Mech. 153, 461-477.

Sommerfeld, A. 1964 Lectures on Theoretical Physics, vol. 3: Electrodynamics. Academic, 371 pp.

Spall, M. A. \& McWilliams, J. C. 1992 Rotational and gravitational influences on the degree of balance in the shallow water equations. Geophys. Astrophys. Fluid Dyn. 64, 1-29.

VALLIS, G. K. 1992 Mechanisms and parameterizations of geostrophic adjustment and a variational approach to balanced flow. J. Atmos. Sci. 49, 1144-1160.

VANNeSte, J. \& Vial, F. 1994 Nonlinear wave propagation on a sphere: interaction between Rossby waves and gravity waves; stability of the Rossby waves. Geophys. Astrophys. Fluid Dyn. 76, 121-144.

WARn, T. 1986 Statistical mechanical equilibria of the shallow-water equations. Tellus 38A, 1-11.

Whitham, G. B. 1974 Linear and Nonlinear Waves. John Wiley, 636 pp.

Williams, G. P. 1985 Geostrophic regimes on a sphere and a beta plane. J. Atmos. Sci. 42, 1237-1243.

YEH, K. C. \& Dong, B. 1988 The non-linear interaction of a gravity wave with the vortical modes. J. Atmos. Terr. Phys. 51, 45-50.

Yuan, L. \& Hamilton, K. 1994 Equilibrium dynamics in a forced-dissipative $f$-plane shallow-water system. J. Fluid. Mech. 280, 369-394. 